\title{
Parameter Estimation Versus Homogenization Techniques in Time-Domain Characterization of Composite Dielectrics
}

\author{
H. T. Banks ${ }^{1}$ V. A. Bokil ${ }^{2}$ and N. L. Gibson, ${ }^{3}$ \\ Center For Research in Scientific Computation \\ North Carolina State University \\ Raleigh, N.C. 27695-8205 \\ USA
}

August 22, 2006

\begin{abstract}
We compare an inverse problem approach to parameter estimation with homogenization techniques for characterizing the electrical response of composite dielectric materials in the time domain. We first consider an homogenization method, based on the periodic unfolding method, to identify the dielectric response of a complex material with heterogeneous micro-structures which are described by spatially periodic parameters. We also consider electromagnetic interrogation problems for complex materials assuming multiple polarization mechanisms with distributions of parameters. An inverse problem formulation is devised to determine effective polarization parameters specific to the interrogation problem. We compare the results of these two approaches with the classical Maxwell-Garnett mixing model and a simplified model with a weighted average of parameters. Numerical results are presented for a specific example involving a mixture of ethanol and water (modeled with multiple Debye mechanisms). A comparison between each approach is made in the frequency domain (e.g., Cole-Cole diagrams), as well as in the time domain (e.g., plots of susceptibility kernels).
\end{abstract}

Keywords: Homogenization, Maxwell's equations, electromagnetic interrogation, inverse problems, parameter estimation, complex dielectric materials, distributions of relaxation parameters, Maxwell-Garnett mixing rule.

\footnotetext{
${ }^{1}$ email: htbanks@ncsu.edu

${ }^{2}$ email: vabokil@ncsu.edu

3 email: ngibson@ncsu.edu
} 


\section{Introduction}

In this paper, we consider composite materials that are mixtures of linear, isotropic and temporally dispersive dielectrics. Composite materials are often used in industry as they have better properties (e.g., electrical, structural) as compared with their constituents. The dielectric properties of mixtures can differ significantly from the individual materials from which they are composed. We compare three different time-domain approaches to characterizing the electrical response of composite dielectrics. We also consider the approach of using the weighted average of the model parameters from each of the constituent materials.

First we consider an homogenization method to identify the dielectric response of a material presenting heterogeneous micro-structures which are described by spatially periodic parameters. When such composite materials are subjected to electromagnetic fields generated by currents of varying frequencies, and the period of the structure is small compared to the wavelength, the spatially dependent coefficients in Maxwell's equations oscillate rapidly. These spatially oscillating coefficients are difficult to treat numerically in simulations. In the process of homogenization the rapidly oscillating coefficients are replaced with new, effective constant (in space) coefficients. The primary objective of homogenization is to replace a system with periodically varying spatial coefficients by a limiting homogeneous system that facilitates computation. The approach that we take here is based on the periodic unfolding method (and its application to homogenization of Maxwell's equations) as presented in $[1,6,8]$.

In a second method we consider a parameter estimation problem which, rather than producing a limiting system with effective coefficients, chooses effective coefficients for a simplified system that best matches the desired output from a specific problem formulation. In particular, we assume an interrogation problem with a given source signal and known geometry. The data to be fit in a least squares sense is a simulation of the complex material using a model involving a distribution of polarization mechanisms as described in [4].

We compare the homogenization and parameter identification technique with results obtained using the Maxwell-Garnett mixing model [15] of a two-phase mixture wherein the inclusions are embedded in a matrix material in the form of spheres (in 3D) or circular disks (in 2D) [14]. We also compare the various techniques mentioned above to a single polarization model which uses the weighted average of the dielectric parameters of the two different constituents of the composite material.

The outline of this paper is as follows. In Section 2, we describe Maxwell's equations and corresponding constitutive relations which govern the evolution of the electromagnetic field in a temporally dispersive media. This is the framework for the various techniques mentioned above.

In Section 3 we present an outline of an homogenization approach, based on the periodic unfolding method, for calculating effective parameters of composite media. Using this approach we can calculate the high frequency permittivity of the composite dielectric as well as a susceptibility function for discrete time values. In this manner we obtain only time-domain data. However, for ease of comparison with frequency domain homogenization methods, we can calculate the complex permittivity of the mixture by performing a discrete Fourier transformation of the susceptibility function data.

In Section 4 we introduce the parameter estimation method based on an inverse problem formu- 
lation. Given data, and a proposed model for simulating an approximation to the data, the inverse problem formulation allows one to determine the model parameters which best match the data in the least squares sense. In the case where the proposed model uses a single polarization mechanism with scalar parameters, the inverse problem method produces effective model parameters similar to an homogenization method. However, the approach is sufficiently general to return a distribution of parameters and/or mechanisms (see [4]). Furthermore, this parameter estimation method is capable of using either simulated or real data in the inverse problem. The method makes no assumption on the geometry of the mixture, rather it assumes a perfectly homogeneous composite. However, as this approach does use information about the interrogating fields, unlike the homogenization methods, it can be tailored to specific nondestructive evaluation (NDE) applications with narrow frequency bands.

The Maxwell-Garnett mixing model presented in Section 5 is also an homogenization type method, but in order to distinguish it from the method described in Section 3, we will refer to it only as a mixing rule. It can be used to calculate an effective complex permittivity of the composite in the frequency domain as well as the susceptibility function values in the time domain. In the Maxwell-Garnett model, it is assumed that one of the components of the mixture exists as spherical (3D) or circular (2D) inclusions inside a background material (ellipsoidal inclusions can also be considered). The homogenization method assumes that the composite has a periodic microstructure where one of the components in the mixture exists as inclusions of a certain geometry inside a background matrix made up of the other component of the mixture. However, unlike the Maxwell-Garnett rule, the geometry of the micro-structure is not limited to circular or spherical geometry. For a proper comparison, in this paper we do assume a circular geometry for the micro-structure. For both the homogenization method based on periodic unfolding as well as the Maxwell-Garnett model, knowledge of the volume proportions of the components of the mixture is needed in order to calculate effective parameters of this composite dielectric.

In Section 6, we present comparisons of these three techniques, along with a simple model involving the weighted average of the dielectric parameters from each constituent material. Time domain comparisons include plots of the susceptibility function, while the frequency domain comparisons involve various plots of the complex permittivity. Finally, we conclude with a summary of results and a discussion of the advantages and disadvantages of the different techniques in Section 8.

\section{Maxwell's Equations in a Dispersive Medium}

We employ Maxwell's equations for a linear and isotropic medium in a form that includes terms for the electric polarization given by

$$
\left\{\begin{array}{l}
\frac{\partial \mathbf{D}}{\partial t}-\nabla \times \mathbf{H}=\mathbf{J} \text { in }(0, T) \times \Omega \\
\frac{\partial \mathbf{B}}{\partial t}+\nabla \times \mathbf{E}=\mathbf{0} \text { in }(0, T) \times \Omega \\
\mathbf{E} \times \mathbf{n}=\mathbf{0} \text { on }(0, T) \times \partial \Omega \\
\mathbf{E}(0, \mathbf{x})=\mathbf{0}, \mathbf{H}(0, \mathbf{x})=\mathbf{0} \text { in } \Omega
\end{array}\right.
$$


along with zero Gauss divergence laws $(\nabla \cdot \mathbf{D}=0, \nabla \cdot \mathbf{B}=0$ in $(0, T) \times \Omega)$. Here $\Omega \subset \mathbb{R}^{n}, n=1,2,3$. The vector valued functions $\mathbf{E}$ and $\mathbf{H}$ represent the strengths of the electric and magnetic fields, respectively, while $\mathbf{D}$ and $\mathbf{B}$ are the electric and magnetic flux densities, respectively. The source current density is given by $\mathbf{J}$. We assume that there are no free electric charges unaccounted for in the electric polarization. We use perfectly conducting boundary conditions on the boundary $\partial \Omega$, with unit outward normal $\mathbf{n}$ and also assign zero initial conditions for all the unknown fields. System (1) is completed by constitutive laws that embody the behavior of the material in response to the electromagnetic fields. These are given in $(0, T) \times \Omega$ in the form

$$
\left(\begin{array}{l}
\mathbf{D}(t, \mathbf{x})=\epsilon_{0} \epsilon_{r}(\mathbf{x}) \mathbf{E}(t, \mathbf{x})+\mathbf{P}(t, \mathbf{x}) \\
\mathbf{B}(t, \mathbf{x})=\mu_{0} \mathbf{H}(t, \mathbf{x})
\end{array}\right.
$$

where $\epsilon_{0}$, and $\mu_{0}$ are the permittivity and the permeability of free space, respectively, $\epsilon_{r}$ is the relative permittivity of the medium under investigation and $\mathbf{P}$ is the media's macroscopic electric polarization. For the media that is of interest to us, we have neglected magnetic effects and we have assumed zero conductivity. To describe the behavior of the media's macroscopic electric polarization $\mathbf{P}$, we employ a general integral representation model in which the polarization explicitly depends on the past history of the electric field. This convolution model is sufficiently general to include microscopic polarization mechanisms such as dipole or orientational polarization as well as ionic and electronic polarization and other frequency dependent polarization mechanisms. The resulting constitutive law can be given in terms of a susceptibility kernel $g$, also known as the dielectric response function (DRF), by

$$
\mathbf{P}(t, \mathbf{x})=g \star \mathbf{E}(t, \mathbf{x})=\int_{0}^{t} g(t-s, \mathbf{x}) \mathbf{E}(s, \mathbf{x}) \mathrm{d} \mathbf{s} .
$$

\subsection{The Debye Model of Orientational Polarization}

The constitutive law (2)-(3) is sufficiently general to include models based on differential equations and systems of differential equations or delay differential equations whose solutions can be expressed through fundamental solutions (in general variation-of-parameters representations)-see [3] for details. A number of known polarization laws can be readily treated. The choice of the kernel function

$$
g(t, \mathbf{x})=\frac{\epsilon_{0}\left(\epsilon_{s}(t, \mathbf{x})-\epsilon_{\infty}(t, \mathbf{x})\right)}{\tau(t, \mathbf{x})} \mathrm{e}^{-t / \tau(t, \mathbf{x})},(t, \mathbf{x}) \in(0, T) \times \Omega
$$

in the dielectric corresponds to the differential equation of the Debye Model for orientational or dipolar polarization given by

$$
\begin{aligned}
\tau(t, \mathbf{x}) \mathbf{P}_{t}(t, \mathbf{x})+\mathbf{P}(t, \mathbf{x}) & =\epsilon_{0}\left(\epsilon_{s}(t, \mathbf{x})-\epsilon_{\infty}(t, \mathbf{x})\right) \mathbf{E}(t, \mathbf{x}) \\
\mathbf{D}(t, \mathbf{x}) & =\epsilon_{0} \epsilon_{\infty}(t, \mathbf{x}) \mathbf{E}(t, \mathbf{x})+\mathbf{P}(t, \mathbf{x})
\end{aligned}
$$

Here $\epsilon_{s}$ is the static relative permittivity. The presence of instantaneous polarization is accounted for in this case by the coefficient $\epsilon_{r}=\epsilon_{\infty}$ in the electric flux equation. The remainder of the electric polarization is seen to be a decaying exponential with relaxation parameter $\tau$, driven by the electric field, less the part included in the instantaneous polarization. This model was first proposed by Debye [10] to model the behavior of materials that possess permanent dipole moments. The magnitude of the polarization term $\mathbf{P}$ represents the degree of alignment of these individual 
moments. The choice of coefficients in (5) gives a physical interpretation to $\epsilon_{s}$ and $\epsilon_{\infty}$ as the relative permittivities of the medium in the limit of the static field and very high frequencies, respectively. In the static case, we have $\mathbf{P}_{t}=0$, so that $\mathbf{P}=\epsilon_{0}\left(\epsilon_{s}-\epsilon_{\infty}\right) \mathbf{E}$ and $\mathbf{D}=\epsilon_{s} \epsilon_{0} \mathbf{E}$. For very high frequencies, $\tau \mathbf{P}_{t}$ dominates $\mathbf{P}$ so that $\mathbf{P} \approx 0$ and $\mathbf{D}=\epsilon_{\infty} \epsilon_{0} \mathbf{E}$.

\section{Homogenization Model in Two Dimensions}

We assume that our problem possesses uniformity in the spatial direction $x_{2}$, so that all derivatives with respect to $x_{2}$ are assumed to be zero. In this case Maxwell's equations decouple into two different modes, the transverse electric (TE) and transverse magnetic (TM) modes. Here, we are interested in the TE mode for which the electric field has two nontrivial components, $\mathbf{E}=$ $\left(E_{x_{1}}, E_{x_{3}}\right)^{T}$, and one nontrivial component $H=H_{x_{2}}$ of the magnetic field. Similarly, the flux densities are $\mathbf{D}=\left(D_{x_{1}}, D_{x_{3}}\right)^{T}$, and $B=B_{x_{2}}$. The system then reduces to vectors depending on two variables, $x_{1}$ and $x_{3}$.

Let $\Omega$ now be a subset of $\mathbb{R}^{2}$ with $\mathbf{x}=\left(x_{1}, x_{3}\right) \in \mathbb{R}^{2}$. Let us define the vector of fields

$$
\mathbf{u}=\left(\mathbf{u}_{1}^{T}, u_{2}\right)^{T}=\left(\mathbf{E}^{T}, H\right)^{T} \in W^{1,1}\left(0, T ; H^{1}\left(\Omega ; \mathbb{R}^{3}\right)\right),
$$

and the operator

$$
\mathbf{L u}(t, \mathbf{x})=\left(\begin{array}{c}
\mathbf{D}(t, \mathbf{x}) \\
B(t, \mathbf{x})
\end{array}\right)
$$

which from (2)-(3) can be written as

$$
\mathbf{L}^{\mathrm{TE}} \mathbf{u}(t, \mathbf{x})=\mathbf{A}^{\mathrm{TE}}(\mathbf{x}) \mathbf{u}(t, \mathbf{x})+\int_{0}^{t} \mathbf{C}^{\mathrm{TE}}(t-s, \mathbf{x}) \mathbf{u}(s, \mathbf{x}) \mathrm{d} s .
$$

The two $3 \times 3$ coefficient matrices in (9) are defined as

$$
\mathbf{A}^{\mathrm{TE}}=\left[\begin{array}{cc}
\mathbf{A}_{11}^{\mathrm{TE}} & 0 \\
\mathbf{0}_{2} & \mu_{0}
\end{array}\right] ; \quad \mathbf{C}^{\mathrm{TE}}=\left[\begin{array}{cc}
\mathbf{C}_{11}^{\mathrm{TE}} & 0 \\
\mathbf{0}_{2} & 0
\end{array}\right],
$$

with

$$
\mathbf{A}_{11}^{\mathrm{TE}}(\mathbf{x})=\left[\begin{array}{cc}
\epsilon_{0} \epsilon_{r}(\mathbf{x}) & 0 \\
0 & \epsilon_{0} \epsilon_{r}(\mathbf{x})
\end{array}\right] ; \quad \mathbf{C}_{11}^{\mathrm{TE}}(t, \mathbf{x})=\left[\begin{array}{cc}
g(t, \mathbf{x}) & 0 \\
0 & g(t, \mathbf{x})
\end{array}\right],
$$

where in the above definitions $\mathbf{0}_{2}$ is the $2 \times 2$ matrix of zeros. Next, we define the Maxwell curl operator $\mathbf{M}^{\mathrm{TE}}$ as

$$
\mathbf{M}^{\mathrm{TE}} \mathbf{u}(t, \mathbf{x})=\mathbf{M}^{\mathrm{TE}}\left(\begin{array}{c}
\mathbf{E}(t, \mathbf{x}) \\
H(t, \mathbf{x})
\end{array}\right)
$$

where the operator $\mathbf{M}^{\mathrm{TE}}$ can be expressed as the matrix operator

$$
\left(\begin{array}{ccc}
0 & 0 & -\partial_{x_{3}} \\
0 & 0 & \partial_{x_{1}} \\
\partial_{x_{3}} & -\partial_{x_{1}} & 0
\end{array}\right)
$$


Thus Maxwell's equation can be rewritten in the form

$$
\left\{\begin{array}{l}
\text { (i) } \frac{d}{d t} \mathbf{L}^{\mathrm{TE}} \mathbf{u}=\mathbf{M}^{\mathrm{TE}} \mathbf{u}+\mathbf{J} \text { in }(0, T) \times \Omega, \\
\text { (ii) } \mathbf{u}(0, \mathbf{x})=\mathbf{0} \text { in } \Omega, \\
\text { (iii) } \mathbf{u}_{1}(t, \mathbf{x}) \times \mathbf{n}(\mathbf{x})=\mathbf{0} \text { on }(0, T) \times \partial \Omega,
\end{array}\right.
$$

where $\mathbf{L}^{\mathrm{TE}}$ is the operator associated with the constitutive law (9), and $\mathbf{M}^{\mathrm{TE}}$ is the Maxwell operator (12).

We assume that the structure that occupies the domain $\Omega$ entails periodic micro-structures leading to matrices $\mathbf{A}^{\mathrm{TE}}, \mathbf{B}^{\mathrm{TE}}$ and $\mathbf{C}^{\mathrm{TE}}$ with spatially oscillatory coefficients. Specifically, we will assume that $\epsilon_{r}$ and $g$ are rapidly oscillating spatial functions.
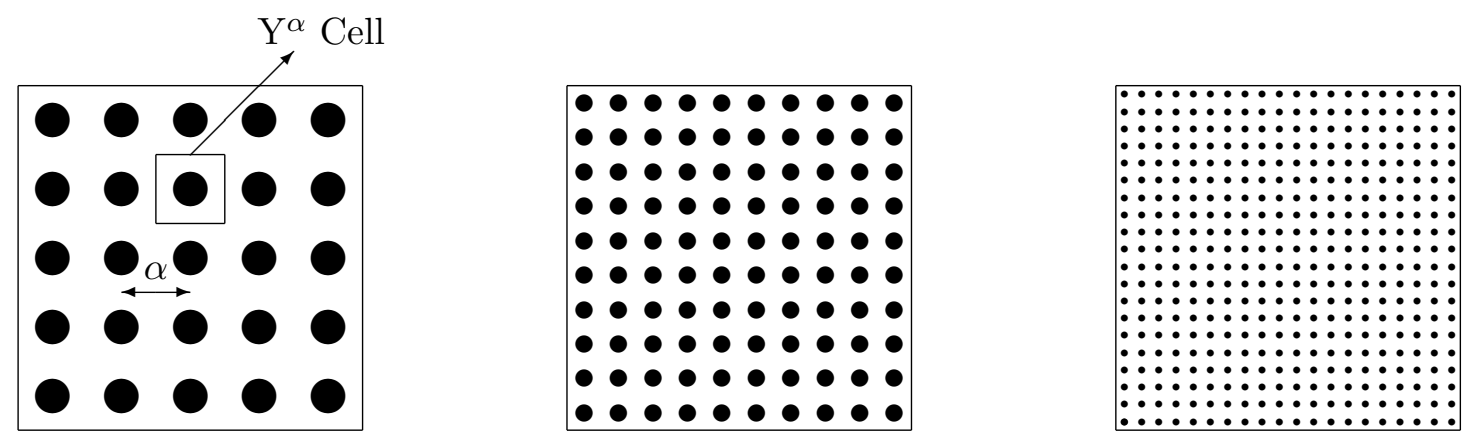

$\alpha \downarrow$

Figure 1: Periodic composite material presenting a circular micro-structure with periodicity $\alpha$. The figure shows $\alpha$ decreasing from left to right.

\subsection{The Homogenized Solution}

The outline presented in this section is based on results from $[1,6]$. We denote by $Y^{\alpha}$ the reference cell of the periodic structure that occupies $\Omega \subset \mathbb{R}^{2}$ (see Figure 1 ). Let $\mathbf{x}, \mathbf{y} \in \mathbb{R}^{2}$ with $\mathbf{x}=\left(x_{1}, x_{3}\right)$ and $\mathbf{y}=\left(y_{1}, y_{3}\right)$. We will use $\mathbf{x} \in \mathbb{R}^{2}$ for points on the macro scale, and $\mathbf{y} \in \mathbb{R}^{2}$ for points on the micro scale (reference cell). Since we assume uniformity in the $x_{2}$-direction, we may have as before all derivatives with respect to $x_{2}$ (or $y_{2}$ in the micro-scale) zero. In this case the homogenized solution $\mathbf{u}=\left(E_{x_{1}}, E_{x_{3}}, H_{x_{2}}\right)^{T}$ for the TE mode appears in a formal asymptotic expansion, or two-scale expansion (see [1]), given using a corrector term $\bar{u}(t, \mathbf{x}, \mathbf{y})$ by

$$
\begin{aligned}
E_{x_{1}}^{\alpha} & =E_{x_{1}}+\partial_{y_{1}} \bar{u}(t, \mathbf{x}, \mathbf{y})+\ldots, \\
E_{x_{3}}^{\alpha} & =E_{x_{3}}+\partial_{y_{3}} \bar{u}(t, \mathbf{x}, \mathbf{y})+\ldots, \\
H_{x_{2}}^{\alpha} & =H_{x_{2}},
\end{aligned}
$$

where the electromagnetic field $\mathbf{u}^{\alpha}=\left(E_{x_{1}}^{\alpha}, E_{x_{3}}^{\alpha}, H_{x_{2}}^{\alpha}\right)^{T}$ is the solution to an evolution problem of type (14) on a domain $\Omega$ with a micro-structure of periodicity $\alpha$. Hence the homogenized electric 
field solution for the TE mode appears in the formal expansion

$$
\mathbf{E}^{\alpha}=\mathbf{E}+\nabla_{\mathbf{y}} \bar{u}(t, \mathbf{x}, \mathbf{y})+\ldots
$$

Since we are assuming uniformity in the $x_{2}$ direction, there is no correction of the magnetic field solution.

The solution of the first corrector term $\bar{u} \in W^{2,1}\left(0, T ; H_{\text {per }}^{1}(Y ; \mathbb{R})\right)$, from the two scale expansion, depends on the calculation of two corrector sub-terms $\bar{w}_{k}^{A} \in H_{\text {per }}^{1}(Y ; \mathbb{R})$ and $\bar{w}_{k} \in$ $W^{1,1}\left(0, T ; H_{\mathrm{per}}^{1}(Y ; \mathbb{R})\right)$, which are solutions to the corrector equations

$$
\left\{\begin{array}{l}
(\mathrm{i}) \int_{Y} \mathbf{A}_{11}^{\mathrm{TE}}(\mathbf{y}) \nabla_{\mathbf{y}} \bar{w}_{k}^{A}(\mathbf{y}) \cdot \nabla_{\mathbf{y}} \bar{v}(\mathbf{y}) \mathrm{d} \mathbf{y}=-\int_{Y} \mathbf{A}_{11}^{\mathrm{TE}}(\mathbf{y}) \mathbf{e}_{k} \cdot \nabla_{\mathbf{y}} \bar{v}(\mathbf{y}) \mathrm{d} \mathbf{y} \\
(\mathrm{ii}) \int_{Y} \mathbf{A}_{11}^{\mathrm{TE}}(\mathbf{y}) \nabla_{\mathbf{y}} \bar{w}_{k}(t, \mathbf{y}) \cdot \nabla_{\mathbf{y}} \bar{v}(\mathbf{y}) \mathrm{d} \mathbf{y} \\
\quad+\int_{Y} \int_{0}^{t} \mathbf{C}_{11}^{\mathrm{TE}}(t-s, \mathbf{y}) \nabla_{\mathbf{y}} \bar{w}_{k}(s, \mathbf{y}) \mathrm{ds} \cdot \nabla_{\mathbf{y}} \bar{v}(\mathbf{y}) \mathrm{d} \mathbf{y} \\
=-\int_{Y} \mathbf{C}_{11}^{\mathrm{TE}}(t, \mathbf{y})\left\{\mathbf{e}_{k}+\nabla_{\mathbf{y}} \bar{w}_{k}^{A}(\mathbf{y})\right\} \cdot \nabla_{\mathbf{y}} \bar{v}(\mathbf{y}) \mathrm{d} \mathbf{y}
\end{array}\right.
$$

for all $\bar{v} \in H_{\text {per }}^{1}(Y), k=1,2$. The vectors $\mathbf{e}_{k}, k=1,2$ are the basis vectors in $\mathbb{R}^{2}$ with $\mathbf{e}_{1}=(1,0)^{T}$, $\mathbf{e}_{2}=(0,1)^{T}$. The gradient operator in this case is $\nabla_{\mathbf{y}}=\left(\partial_{y_{1}}, \partial_{y_{3}}\right)^{T}$. The first corrector term $\overline{\mathbf{u}}$ can now be calculated as

$$
\bar{u}(t, \mathbf{x}, \mathbf{y})=\overline{\mathbf{w}}^{A}(\mathbf{y}) \mathbf{E}(t, \mathbf{x})+\int_{0}^{t} \overline{\mathbf{w}}(t-s, \mathbf{y}) \mathbf{E}(s, \mathbf{x}) \mathrm{ds},
$$

where $\overline{\mathbf{w}}^{A} \in \mathbb{R}^{1 \times 2}$ with components $\left\{\bar{w}_{k}^{A}\right\}_{k=1}^{2}$. Similarly $\overline{\mathbf{w}} \in \mathbb{R}^{1 \times 2}$ with components $\left\{\bar{w}_{k}\right\}_{k=1}^{2}$.

Once we have solved for these corrector terms, we can then construct the homogenized matrices from

$$
\left\{\begin{array}{l}
\text { (i) } \quad\left(\mathcal{A}_{11}^{\mathrm{TE}}\right)_{k}=\int_{Y} \mathbf{A}_{11}^{\mathrm{TE}}(\mathbf{y})\left\{\mathbf{e}_{k}+\nabla_{\mathbf{y}} \bar{w}_{k}^{A}(\mathbf{y})\right\} \mathrm{d} \mathbf{y} \\
\left(\text { ii) } \quad\left(\mathcal{C}_{11}^{\mathrm{TE}}\right)_{k}(t)=\int_{Y} \mathbf{C}_{11}^{\mathrm{TE}}(t, \mathbf{y})\left\{\mathbf{e}_{k}+\nabla_{\mathbf{y}} \bar{w}_{k}^{A}(\mathbf{y})\right\} \mathrm{d} \mathbf{y}+\int_{Y} \mathbf{A}_{11}^{\mathrm{TE}}(\mathbf{y}) \nabla_{\mathbf{y}} \bar{w}_{k}(t, \mathbf{y}) \mathrm{d} \mathbf{y}\right. \\
\quad+\int_{Y} \int_{0}^{t} \mathbf{C}_{11}^{\mathrm{TE}}(t-s, \mathbf{y}) \nabla_{\mathbf{y}} \bar{w}_{k}(s, \mathbf{y}) \mathrm{ds} \mathrm{d} \mathbf{y}
\end{array}\right.
$$

where, $\left(\mathcal{A}_{11}^{\mathrm{TE}}\right)_{k}$ and $\left(\mathcal{C}_{11}^{\mathrm{TE}}\right)_{k}$ are the $k$ th columns of the $2 \times 2$ matrices $\mathcal{A}_{11}^{\mathrm{TE}}$ and $\mathcal{C}_{11}^{\mathrm{TE}}$, respectively, and the homogenized matrices are given by

$$
\mathcal{A}^{\mathrm{TE}}=\left[\begin{array}{cc}
\mathcal{A}_{11}^{\mathrm{TE}} & 0 \\
0 & \mu_{0}
\end{array}\right], \quad \mathcal{C}^{\mathrm{TE}}=\left[\begin{array}{cc}
\mathcal{C}_{11}^{\mathrm{TE}} & 0 \\
0 & 0
\end{array}\right] .
$$

The corresponding system of equations in the TE mode are

$$
\left\{\begin{array}{l}
\text { (i) } \frac{d}{d t} \mathcal{L}^{\mathrm{TE}} \mathbf{u}=\mathbf{M}^{\mathrm{TE}} \mathbf{u}+\mathbf{J}_{s}^{\mathrm{TE}} \text { in }(0, T) \times \Omega, \\
\text { (ii) } \mathbf{u}(0, \mathbf{x})=\mathbf{0} \text { in } \Omega, \\
\text { (iii) } \mathbf{u}_{1}(t, \mathbf{x}) \times \mathbf{n}(\mathbf{x})=\mathbf{0} \text { on }(0, T) \times \partial \Omega,
\end{array}\right.
$$


where $\mathbf{u}=\left(E_{x_{1}}, E_{x_{3}}, H_{x_{2}}\right)^{T}, \mathbf{n}=\left(n_{x_{1}}, n_{x_{3}}\right)^{T}$ is the unit outward normal vector to $\partial \Omega$, and the operator $\mathcal{L}^{\mathrm{TE}}$ is defined as

$$
\mathcal{L}^{\mathrm{TE}} \mathbf{u}(t, \mathbf{x})=\mathcal{A}^{\mathrm{TE}} \mathbf{u}(t, \mathbf{x})+\int_{0}^{t} \mathcal{C}^{\mathrm{TE}}(t-s) \mathbf{u}(s, \mathbf{x}) \mathrm{ds}
$$

If the initial conditions are nonzero, additional corrector terms and corrector equations are involved, and then there is a supplementary source term $\mathcal{J}^{0}$ that should be introduced in the right side of $(21, \mathrm{i})$. See $[6]$ for more details.

\subsection{Numerical Discretization for the Cell Problem}

The spatial discretization of the cell problem can be done using piecewise bilinear finite elements. For the time discretization we use an approach that involves calculating the convolution recursively. Since the susceptibility kernel $g(t, \mathbf{x})$ is exponential in nature for many materials of interest, we can use recursion to compute the discretized time convolution of the susceptibility kernel with the gradient of the corrector terms in the corrector subproblem $(17$,ii) and the construction $(19$,ii). The discrete gradient of the corrector term is assumed to be constant on each equally spaced interval of time $\Delta t$. The details of the method summarized here may be found in [1]. A similar approach known as the recursive convolution (RC) method has been used to compute the discrete convolution terms that appear in Maxwell's equations [12, 13]. We show in [1] that the discrete convolution of all previous field values of the gradient of the corrector terms and the discrete susceptibility function can be reduced to recursive updating of a single vector on each element in the finite element mesh; this involves a matrix vector multiplication at each time step.

For the numerical experiments in this paper we obtain the effective high frequency permittivity as well as time plots of the effective susceptibility kernels for various dielectric mixtures. We do so by solving specific cell problems in the reference cell $Y=[0,1] \times[0,1]$. The simulations are performed on a $51 \times 51$ nodes finite element grid. In the numerical solution for the homogenized model we have approximated circular micro-structures in a staircase fashion. For details see [1]. The results of several experiments will be presented in Section 6 .

\section{Parameter Estimation}

We next formulate an inverse problem for the determination of parameters for a model which describes the dielectric response of complex materials in the context of electromagnetic interrogation. It is well known that, in general, dielectrics are most accurately modeled by a distribution of parameters [7], although using a distribution of parameters in practice is sometimes computationally intensive. Thus there is a need to determine single, effective parameters. The comparison of several ways to do this is the precisely the subject of this paper.

In [4] it was shown that the inverse problem for the actual distribution is well posed. In fact, distributions of mechanisms (e.g., mixtures of Debye and Lorentz materials) could also be handled in the same framework. Using this theory as a foundation, we consider the inverse problem wherein parameters for a simplified model are estimated. The most simplified case of a single mechanism involving a delta distribution of the parameter set results in the determination of effective model parameters as produced in homogenization techniques. However, unlike homogenization methods, the 
inverse problem formulation has the flexibility to use either real data or simulated data obtained by using a suitable forward solve. Further, as the electromagnetic interrogation of an object generally involves a relatively narrow range of frequencies, the inverse problem based parameter estimation is designed to essentially match the dielectric response of the material in the frequency band of interest. This is unlike homogenization methods which do not take the interrogating frequency into account.

\subsection{Distributions of Mechanisms and Parameters}

As before, we consider the electric field $\mathbf{E}$ and the magnetic field $\mathbf{H}$ to be governed by Maxwell's equations in a domain $\mathcal{D}=\Omega_{0} \cup \Omega$ where $\Omega$ contains the dielectric and the ambient $\Omega_{0}$ is treated as a vacuum. (See Figure 2 for a schematic of a sample domain.)

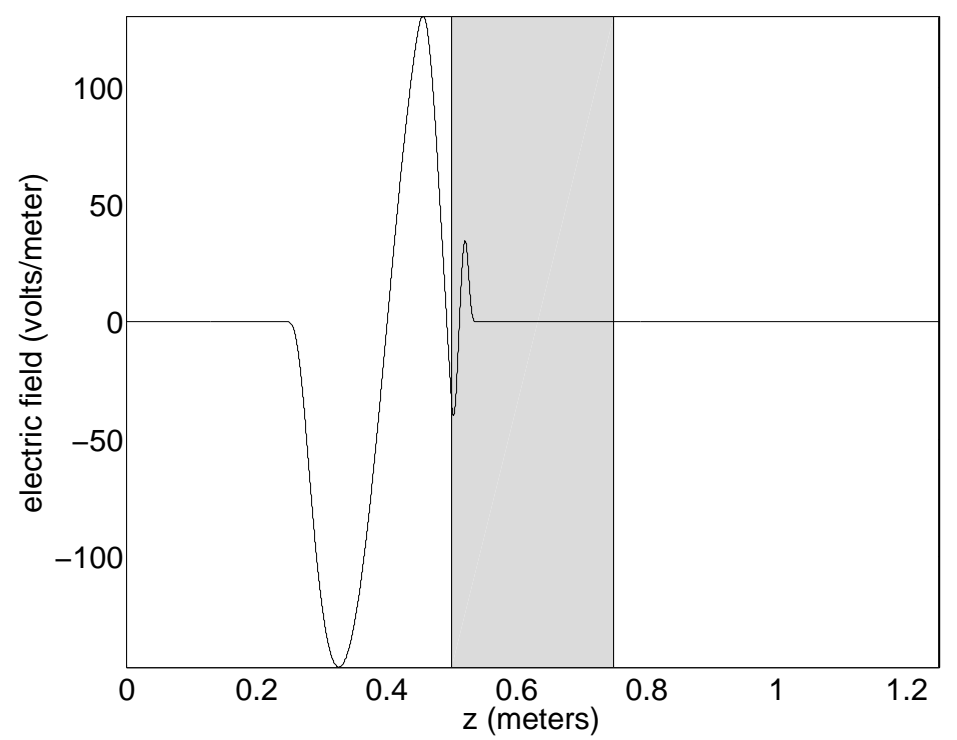

Figure 2: Snapshot of a simulation of a truncated sine wave partially reflecting from and partially penetrating a sample material. The $z$-axis represents a sample $1 \mathrm{D}$ domain.

Our main focus in this section is the dielectric polarization $\mathbf{P}$ which we assume has the general convolution form given in (3). In every practical example (Debye, Lorentz, etc.) DRF's are parameter dependent as well as time (and possibly space) dependent; we represent this as $g=g(t, \mathbf{x} ; \nu)$, where typically $\nu$ contains parameters such as the high frequency limit dielectric permittivity $\epsilon_{\infty}$, the static permittivity $\epsilon_{s}$, and relaxation time $\tau$. An example of an often-used DRF is the Debye model (described in Section 2.1). Allowing for a distribution $F$ of parameters $\nu$ over some admissible set $\mathcal{N}$, we generalize the polarization law (3) to

$$
\mathbf{P}(t, \mathbf{x} ; F)=g \star \mathbf{E}(t, \mathbf{x})=\int_{0}^{t} \int_{\mathcal{N}} g(t-s, \mathbf{x} ; \nu) \mathbf{E}(s, \mathbf{x}) d F(\nu) d s .
$$

We expect to chose $F$ from (or from a subset of) the space $\mathcal{F}=\mathfrak{P}(\mathcal{N})$ of all probability measures $F$ on $\mathcal{N}$. We could further generalize (23) to allow for dielectric materials with multiple mechanisms or multiple DRF's (i.e., heterogeneous molecular structures) by considering a family of possible 
DRF's and distributions over this family as described in [4]. For now we will restrict our efforts to single mechanisms.

\subsection{Inverse Problem Formulation}

In [4] the focus was to determine the parameters of distributions in an inverse problem formulation in order to accurately describe the electrical response of complex materials. In this effort we attempt to find a single set of effective parameters that "best" describe this response for a specific interrogating source. In other words, we are attempting to find the distribution $F$ in the subset of $\mathcal{F}=\mathfrak{P}(\mathcal{N})$ which corresponds to all single delta distributions, even though the data may (and does!) come from simulations involving more than just a single parameter set.

Formally, we consider the Maxwell system (1)-(2) with polarization $\mathbf{P}=\mathbf{P}(t, \mathbf{x} ; F)$ given by (3). Let

$$
\mathbf{z}(t, \mathbf{x} ; F)=\left(\begin{array}{c}
\mathbf{E}(t, \mathbf{x} ; F) \\
\mathbf{H}(t, \mathbf{x} ; F)
\end{array}\right)
$$

with $(t, \mathbf{x}) \rightarrow \mathbf{z}(t, \mathbf{x} ; F)$ mapping from $(0, T) \times \Omega$ to $R^{6}$. We assume we are given data $\overline{\mathbf{d}}=$ $\left\{\mathbf{d}_{i}\right\}_{i=1}^{n}$ corresponding to observations of $\mathcal{C}_{A} \mathbf{z}\left(t_{i}, \cdot ; F\right)$. Here $\mathcal{C}_{A}$ denotes evaluation of one or more components of $\mathbf{E}$ or $\mathbf{H}$ at an antenna $\left\{\mathbf{x}_{A}\right\}$. We use this data to estimate the parameter distribution $F$ in an ordinary least squares (OLS) formulation, seeking to minimize

$$
J(F)=\sum_{i=1}^{n}\left|\mathcal{C}_{A} \mathbf{z}\left(t_{i}, \cdot ; F\right)-\mathbf{d}_{i}\right|^{2},
$$

over some subspace the space $\mathcal{F}=\mathfrak{P}(\mathcal{N})$ of all probability measures $F$ on $\mathcal{N}$.

For this particular effort, we are concerned with comparing the inverse problem based parameter estimation method to other methods of determining effective parameters. Thus, while the synthetic data is generated using the general formulation of the polarization law (23), the forward solves in the inverse problem will be limited to a single polarization mechanism with a single parameter set $\nu$, namely, equation (3).

\subsection{Numerical Results}

As our inverse problem approach does not depend on the inclusion geometry, we may consider a 1-D example as explained in detail in [5]. Restricting to one dimension, and using $D=\epsilon E+P$, we can write Maxwell's equations in second order form as

$$
\mu_{0} \epsilon \ddot{E}+\mu_{0} I_{\Omega} \ddot{P}-E^{\prime \prime}=-\mu_{0} \dot{J}_{s} \text { in } \Omega \cup \Omega_{0}=[0, b],
$$

where $E$ is the $\hat{k}$ or $z$ component of the electric field, and $\epsilon=\epsilon_{0}\left(1+I_{\Omega}\left(\epsilon_{\infty}-1\right)\right)$. Here, $I_{\Omega}$ denotes the indicator function on the dielectric medium $\Omega$. The (now scalar) polarization is still determined by equation (3), however with the vector valued electric field $\mathbf{E}$ replaced by the scalar $E$. We assume zero initial conditions for $E$ and $P$,

$$
P(0, z)=E(0, z)=\dot{E}(0, z)=0 .
$$


Lastly, we have absorbing boundary conditions at $z=0$ and reflecting conditions at $z=b$, namely

$$
\begin{aligned}
{\left[\dot{E}-c E^{\prime}\right]_{z=0} } & =0 \\
E(t, b) & =0
\end{aligned}
$$

where $c^{2}=\frac{1}{\epsilon_{0} \mu_{0}}$.

The numerical method employed involves a linear Finite Element Method for spatial discretization, and a centered difference for the temporal discretization. For details, see [2].

We choose to consider a mixture of two Debye materials, for which the parameter identification procedure will attempt to find parameters for a single Debye model such that the resulting simulations best match, in a least squares sense, the data generated from the mixture. The substance that is being interrogated is a mixture of water and ethanol with volume fractions of $80 \%-20 \%$, $50 \%-50 \%$, and $20 \%-80 \%$. For water, we used the following parameters in a Debye polarization model: $\tau_{1}=1.01 \times 10^{-11}, \epsilon_{s, 1}=80.1$, and $\epsilon_{\infty, 1}=4.9$. The Debye parameters for ethanol are $\tau_{2}=1.2 \times 10^{-10}, \epsilon_{s, 2}=25.1$, and $\epsilon_{\infty, 2}=4.4$.

We assume the interrogating signal to be a single cycle truncated sine curve with carrier frequency $10^{9} \mathrm{~Hz}$. Note that this frequency is below the weighted average relaxation frequency $f=1 / \tilde{\tau}$, with $\tilde{\tau}=\alpha \tau_{1}+(1-\alpha) \tau_{2}$ (here $\alpha$ is the volume fraction of water), and therefore we expect that the optimal single relaxation time should be close to this average value [4]. Further, for simplicity of computations, we take the value of $\epsilon_{\infty}$ in the single Debye model to be the weighted average of mixture, namely $\epsilon_{\infty, e f f}=\alpha \epsilon_{\infty, 1}+(1-\alpha) \epsilon_{\infty, 2}$. This results in a two parameter inverse problem for $\tau$ and $\epsilon_{s}$. The interrogating source and the signal receiver are both located at $z=0$ (see Figure 2). For this test problem, the cost functional is

$$
J\left(\tau, \epsilon_{s}\right)=\sum_{i=1}^{n}\left|E\left(t_{i}, 0 ; \tau, \epsilon_{s}\right)-\mathbf{d}_{i}\right|^{2} .
$$

A surface plot of each of the cost functionals $J$ is displayed in Figures 3-5 for the volume fractions of $80 \%-20 \%, 50 \%-50 \%$, and $20 \%-80 \%$, respectively. The left plot of each figure depicts the range of values between the parameters for ethanol and the parameters for water. The right plot of each figure shows a closer view of the objective function near the optimal solution. The initial starting point used in the optimization method is marked with an "O". This value is simply the weighted average of $\tau$ and $\epsilon_{s}$ over the material. The resulting optimum value from the optimization method is marked with an "X".

Note that in the $80 \%-20 \%$ case there is a large positive peak centered at $\epsilon_{s}=60$. We do not have to resort to taking the absolute value of the electric field to avoid this obstacle to minimization, as described in [4], since our starting point for the optimization method is closer to the minimum than this peak. For the 20\%-80\% case there is a local minimum near $\epsilon_{s}=53$. Fortunately, for these particular parameters, the initial solution is close enough to the global minimum to not converge to the local minimum. It is important to note however, that for other choices of parameters, convergence to the incorrect minimum is a very real concern.

For the optimization method, we use a Levenberg-Marquardt method with implicit filtering. As the cost functional is significantly more sensitive to the $\epsilon_{s}$ direction than the $\tau$ direction, taking a Newton step in both directions at the same time generally results in an increased value of the cost 

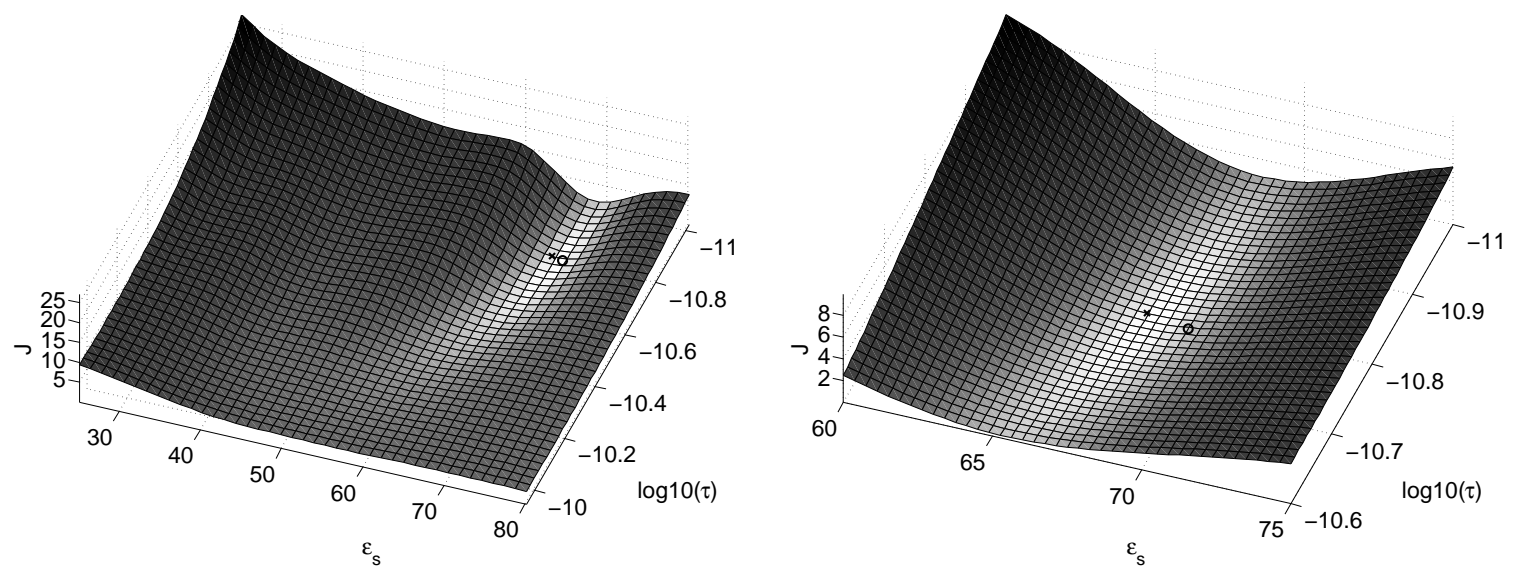

Figure 3: The left plot depicts the objective function for the parameter identification problem versus the $\log$ of $\tau$ and $\epsilon_{s}$ using a volume fraction of $80 \%-20 \%$ water to ethanol. The right plot presents a closer view of the objective function, with the initial value marked with an "O" and the optimal value marked with an "X".

function. Rather than increasing the Levenberg-Marquardt parameter immediately (i.e., performing a pull-back on the line-search which results in a shorter step), we test the possibility that taking just one of the directions will give an actual reduction in the objective function. The addition of these two function evaluations, when the cost is not decreased by the original step, saved approximately $70 \%$ of the pull-backs on line-searches versus the traditional Levenberg-Marquardt method, and led to faster convergence. Table 1 contains the initial values of $\log (\tau), \epsilon_{s}$ and $J$, as well as the optimized values for each case of differing volume fractions (where $\alpha$ is the percentage of water).

Table 1: Initial values and values resulting from Levenberg-Marquardt optimization of the waterethanol mixture using the volume fractions of $80 \%-20 \%, 50 \%-50 \%$, and $20 \%-80 \%$ interrogated at $f=10^{9} \mathrm{~Hz}$.

\begin{tabular}{|c|ccc|}
\hline$\alpha=.8$ & $\log (\tau)$ & $\epsilon_{s}$ & $J$ \\
\hline Initial & -10.7807 & 69.1 & 0.350694 \\
Optimal & -10.7933 & 67.5529 & 0.0122314 \\
\hline$\alpha=.5$ & $\log (\tau)$ & $\epsilon_{s}$ & $J$ \\
\hline Initial & -10.4582 & 52.6 & 2.48618 \\
Optimal & -10.5759 & 48.2837 & 0.182077 \\
\hline$\alpha=.2$ & $\log (\tau)$ & $\epsilon_{s}$ & $J$ \\
\hline Initial & -10.1358 & 36.1 & 2.92976 \\
Optimal & -10.418 & 30.1762 & 1.54837 \\
\hline
\end{tabular}

To see the improvement in the fit-to-data of the optimal value response over that of the weighted average in matching the multiple Debye signal, we plot in Figures 6-8 the resulting simulations and the original data computed using a discrete distribution of two Debye models, for each of the three volume fractions. The inset shows a closer view of the full signal reflected from the back boundary (the only useful information in determining the electrical response in an experiment). 

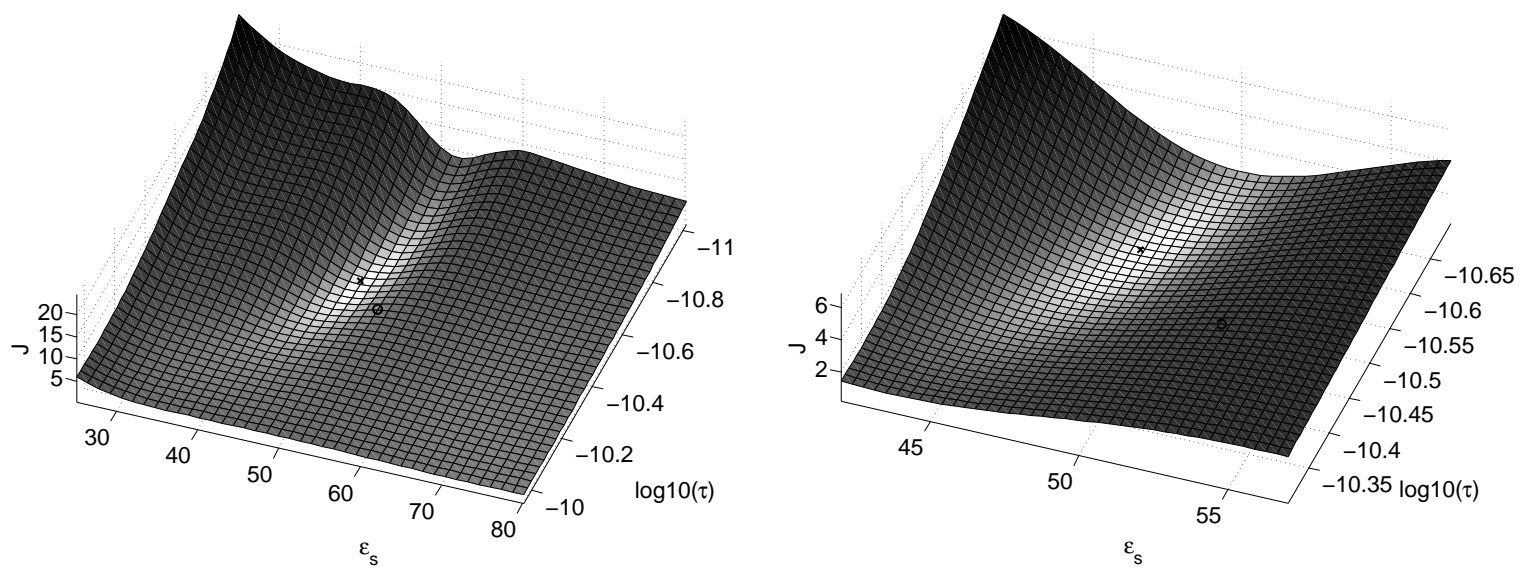

Figure 4: The left plot depicts the objective function for the parameter identification problem versus the $\log$ of $\tau$ and $\epsilon_{s}$ using a volume fraction of $50 \%-50 \%$ water to ethanol. The right plot presents a closer view of the objective function, with the initial value marked with an "O" and the optimal value marked with an "X".

It is clear from each of the figures that the weighted average parameters do not result in signals that match the double Debye data. For the cases $80 \%-20 \%$ and $50 \%-50 \%$, the optimal parameters produce a signal that is very similar to the data, although still distinguishable. However, even for these simple examples it is apparent that no single Debye model can produce a simulation exactly matching the double Debye model. In fact, this conclusion is even more obvious in the $20 \%-80 \%$ case. The optimization method returns this solution as optimum, and the parameter values can be visually verified by referring to the surface plot 5 , but clearly the single Debye simulation does not match the multiple Debye in amplitude nor phase. Still, when an approximate result is sufficient and the savings in computational time important, then the optimal parameters from an inverse problem formulation provide an alternative to modeling a complex material using distributions of parameters and mechanisms.

\section{The Maxwell Garnett Model}

We will compare the homogenization method and the parameter identification technique presented above to the Maxwell-Garnett (MG) model of a two-phase mixture wherein the inclusions are embedded in the environment in the form of spheres (in 3D) or circular disks (in 2D). As in the case of the other techniques, we assume that both the temporally dispersive materials are isotropic, homogeneous and non-magnetic.

A direct time-domain approach that has been developed in [14] expresses the effective permittivity of a mixture as an operator form MG expression that includes the susceptibility kernels and high frequency responses of the component materials. The evaluation of the effective permittivity operator requires the calculation of convolutions and operator inverses. The inverse of the convolution operators encountered in these problems can be solved from Volterra equations of the second kind, which have unique and well behaved solutions. The advantage of the time-domain formulation is that the results are more intuitive physically than those in the frequency domain. When both 

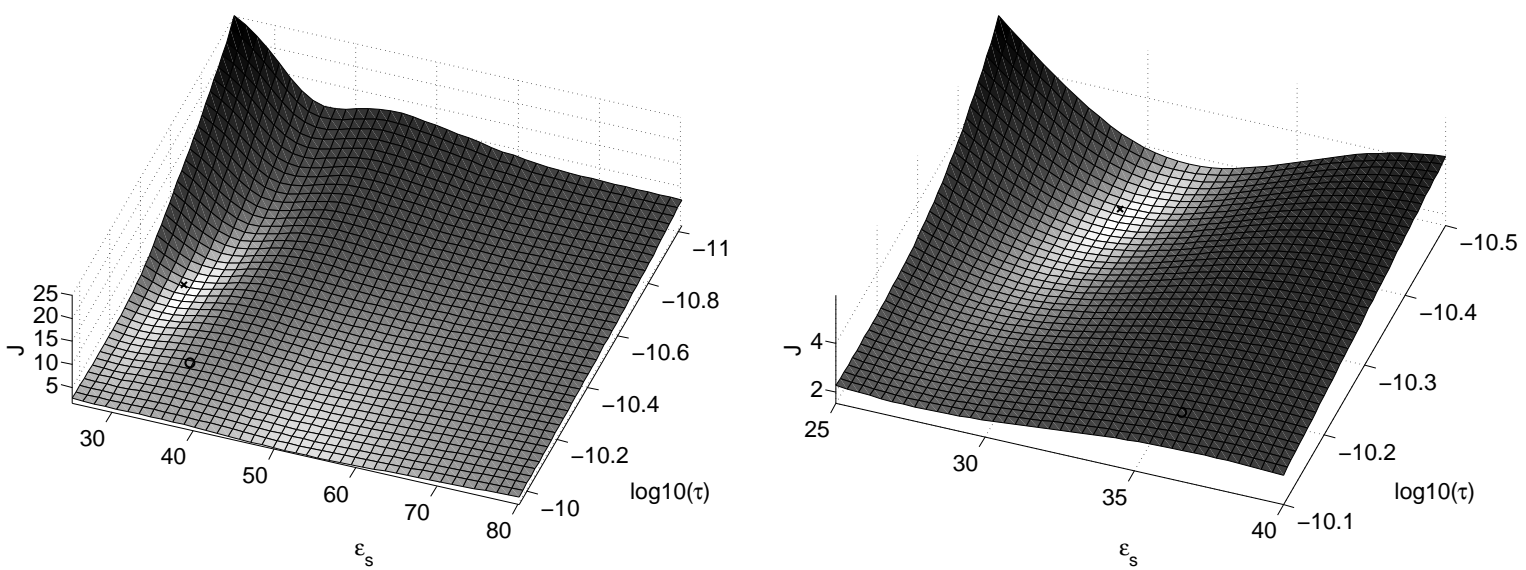

Figure 5: The left plot depicts the objective function for the parameter identification problem versus the $\log$ of $\tau$ and $\epsilon_{s}$ using a volume fraction of $20 \%-80 \%$ water to ethanol. The right plot presents a closer view of the objective function, with the initial value marked with an "O" and the optimal value marked with an "X".

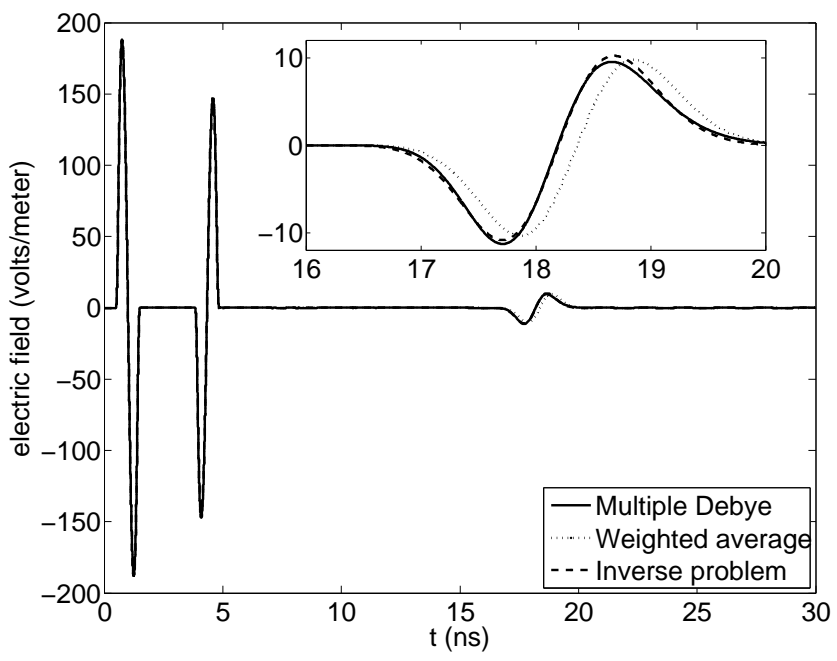

Figure 6: Comparison of multiple Debye data to the forward simulation using the weighted average Debye parameter values and the forward simulation using optimal values from the single Debye inverse problem using a volume fraction of $80 \%-20 \%$.

constituent materials are dispersive, the dispersion of the mixture is in general more complicated than that of the inclusion. Even for the simplest case of Debye inclusions in a Debye background, the effective complex permittivity is neither of Debye nor of Lorentz form, but contains higher powers of the frequency $\omega$ in both the numerator and the denominator.

For the examples of an $80 \%$ water and $20 \%$ alcohol mixture, and a $20 \%-80 \%$ mixture, explicit expressions for the effective susceptibility kernel are given in Sec 5.B.3 of [14]. The analysis in [14] is restricted to the case of spherical (circular in 2D) inclusions. The homogenization technique presented in Section 3, however, does not have this restriction. 


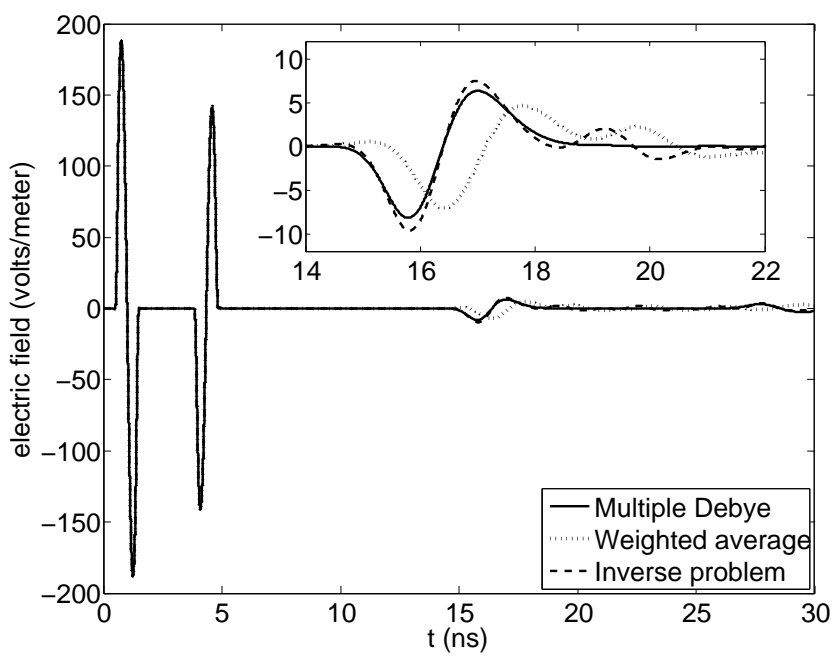

Figure 7: Comparison of multiple Debye data to the forward simulation using the weighted average Debye parameter values and the forward simulation using optimal values from the single Debye inverse problem using a volume fraction of $50 \%-50 \%$.

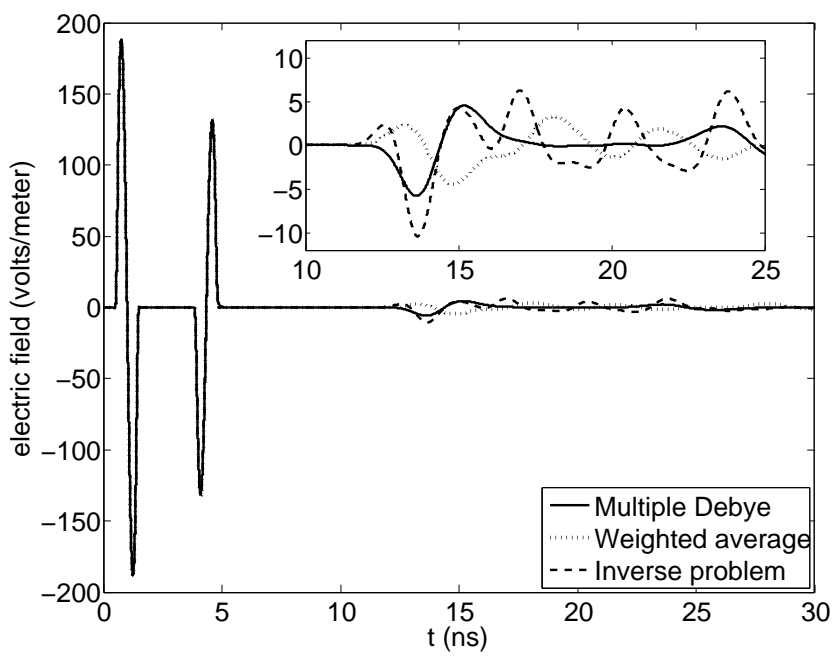

Figure 8: Comparison of multiple Debye data to the forward simulation using the weighted average Debye parameter values and the forward simulation using optimal values from the single Debye inverse problem using a volume fraction of $20 \%-80 \%$.

\section{Comparison of Methods}

We compare the time domain and frequency domain results of each of the methods described above. For the time domain, we plot the DRF used in the convolution definition of the polarization from equation 3. While the homogenization method used here directly produces a dielectric response function defined at specified time points, the inverse problem approach is designed to simply output a single parameter set for use in a Debye polarization model formulation. To compare the two methods in the time domain we may use the fact that the Debye model DRF is given by (4). 
In order to examine the differences in the frequency domain we must compare the resulting complex permittivity. For the Debye polarization model, the (relative) complex permittivity is given by

$$
\epsilon(\omega)=\epsilon_{\infty}+\frac{\epsilon_{s}-\epsilon_{\infty}}{1+i \omega \tau}
$$

However, the homogenization approach used here does not output a complex permittivity directly. One may take the DFT of the DRF to get the permittivity. This presents some computation issues when attempting to plot the permittivity for the entire range of frequencies of interest. Therefore, plots involving the homogenization method are incomplete in some places, and care must be taken in interpreting the results. See Section 7 for a discussion on the convergence of the permittivity curves as the discretization parameters are reduced. In general, the homogenization results tend to match those of the classical Maxwell-Garnett mixing formula. A more efficient approach for frequency domain comparison would be to compute the effective permittivity from the homogenization method within a frequency domain formulation, as described in [6]. However, the main purpose of this effort is time domain comparisons, therefore we only present the frequency domain results to emphasize the difference between the methods. Plots of the real and imaginary parts of the complex permittivity are plotted in the following subsections.

An additional depiction of the complex permittivity is the Cole-Cole diagram. Cole and Cole [9] proposed an empirical function for use in fitting dielectric data. The Debye model presented in Section 2.1 is a special case of this Cole-Cole model. The Cole-Cole function (including Debye) leads to a distinctive semicircular plot in the complex permittivity plane. As the frequency is varied, a plot of the imaginary part of the complex permittivity versus the real part describes a circular arc [11] which among other names is called the Cole-Cole diagram.

\section{1 $80 \%-20 \%$ Volume Fraction}

The left plot of Figure 9 depicts the DRF's for the inverse problem result, the solution from the homogenization approach, as well as the curves for a Debye model of pure water and for pure ethanol. In the right plot of Figure 9 we compare the DRF from the inverse problem and homogenization approaches to the MG mixing rule result and the DRF obtained by using the weighted average of values in the Debye model (4). The inverse problem DRF is nearly indistinguishable from that of the weighted averages; however, the difference between the corresponding outputs from the interrogation simulations was clearly seen in Figure 6.

The real and imaginary parts of the permittivity resulting from the homogenization approach and the inverse problem approach, are plotted in Figure 10, along with the values for pure water and pure ethanol. Both methods have curves that lie between those of the water and ethanol, although more closely aligned with water. However the main difference is that the homogenization approach has a peak in the imaginary part of the permittivity very close to the relaxation frequency of water, whereas the inverse problem formulation seems to have a peak close to the weighted average of relaxation frequencies.

In Figure 11 the real and imaginary parts of the permittivity from the homogenization approach and the inverse problem approach are compared to the values resulting from taking the weighted average of parameters and the Maxwell-Garnett mixing method.

The peaks in the plots of the imaginary parts denote the frequency at which the maximum 

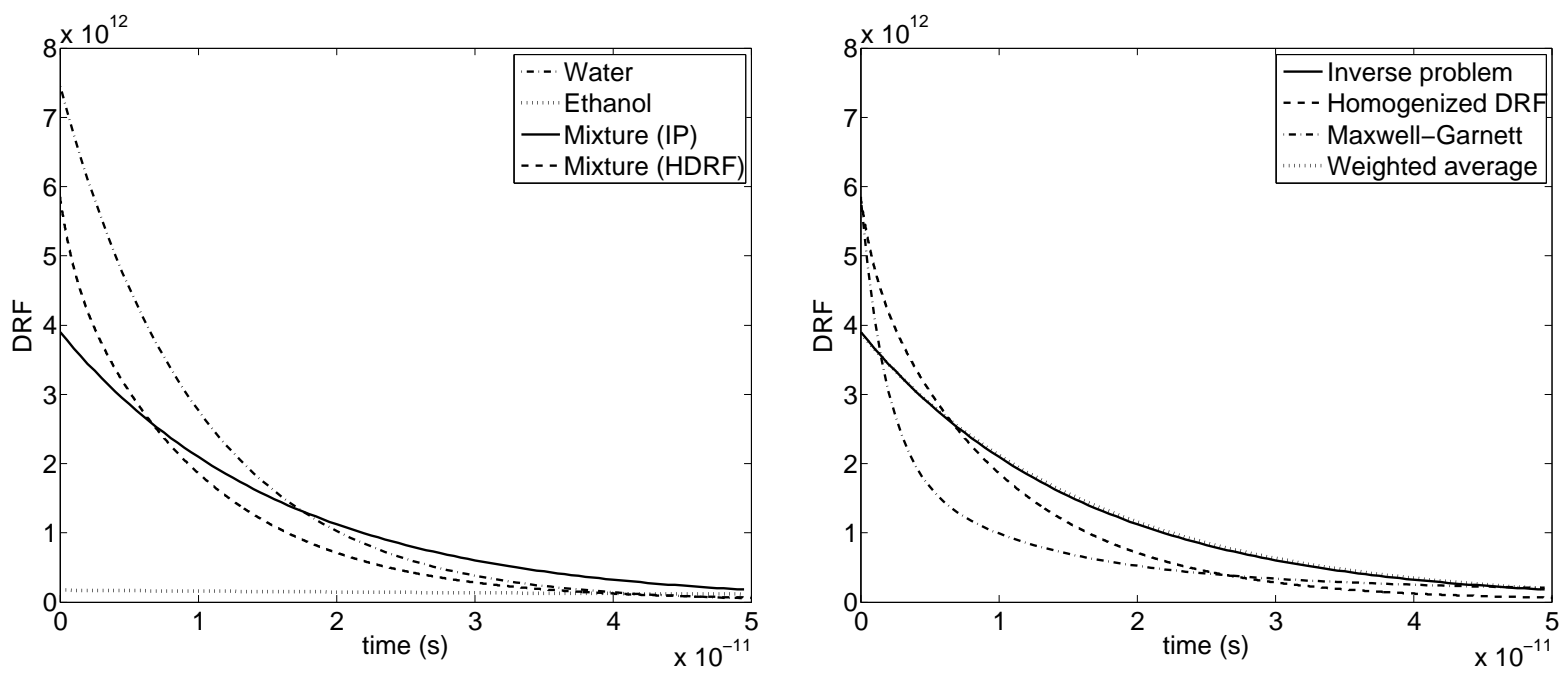

Figure 9: The left side presents a plot of the DRF resulting from the homogenization approach and the inverse problem approach, using a volume fraction of $80 \%-20 \%$, along with the plots for the Debye model of water and ethanol. The right side depicts the same for the homogenization and inverse problem approaches, along with the DRF's computed using the Maxwell-Garnett mixing formula and a weighted average. The DRF of the weighted average approach and the inverse problem method are nearly indistinguishable.
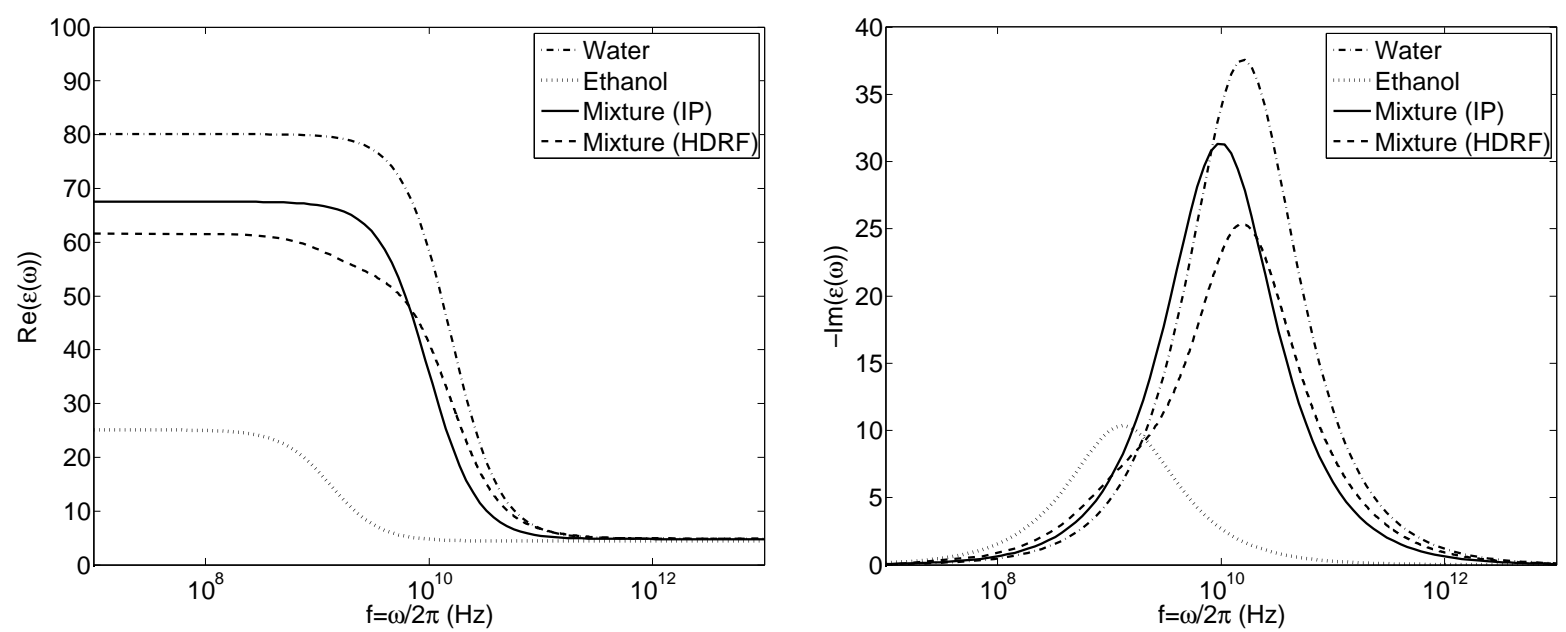

Figure 10: The left (right) graph depicts the real (negative imaginary) part of the permittivity resulting from the homogenized DRF and the inverse problem approach using a volume fraction of $80 \%-20 \%$, along with the values for pure water and pure ethanol.

attenuation of the field in the material occurs, (in the Debye case it is known as the relaxation frequency). Note that both the Maxwell-Garnett and the homogenization methods have peaks very close to that of water. Both the parameter estimation method and the approach using weighted average of parameters result in peaks very close to the weighted average of the relaxation frequencies of water and ethanol. 

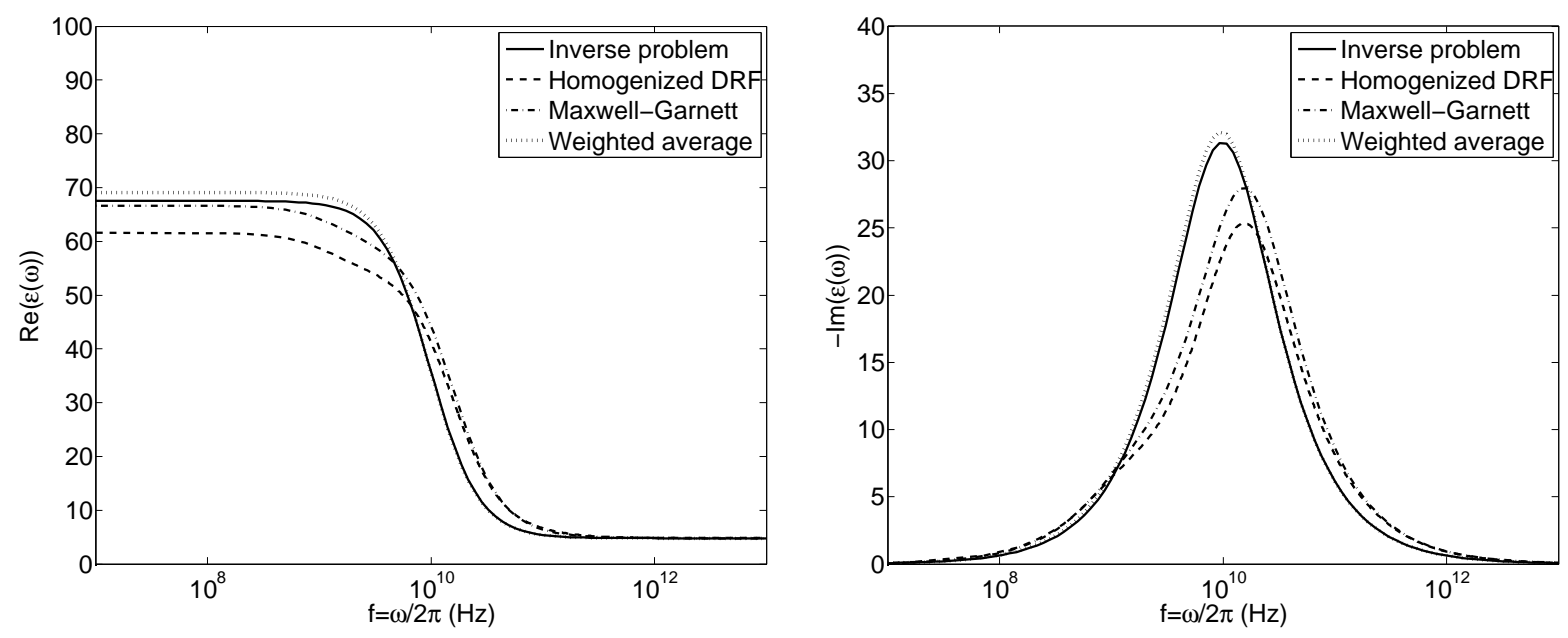

Figure 11: The left (right) graph presents the real (negative imaginary) part of the permittivity resulting from the homogenized DRF and the inverse problem approach, along with the values computed using a weighted average and the Maxwell-Garnett mixing formula, using a volume fraction of $80 \%-20 \%$.
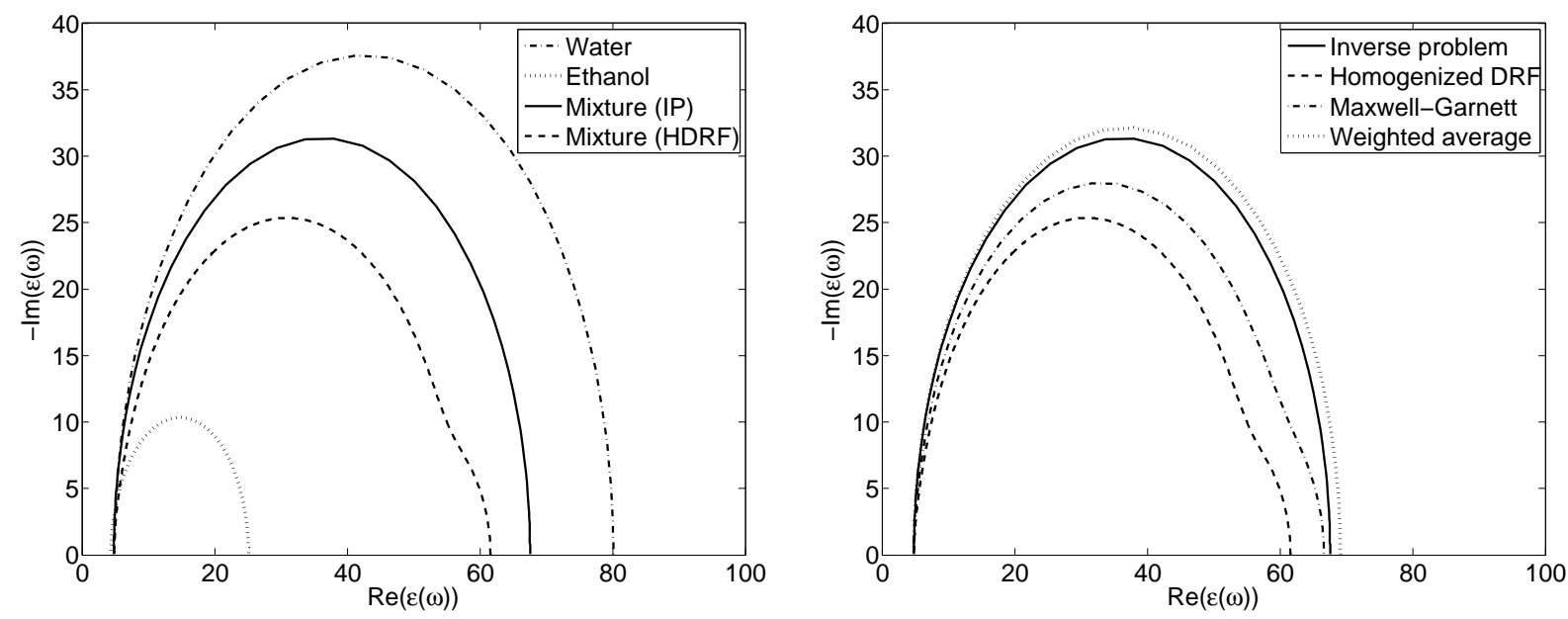

Figure 12: The left plot depicts the Cole-Cole diagram of the complex permittivity resulting from the homogenization approach and the inverse problem approach, along with the values from the Debye model for water and ethanol using a volume fraction of $80 \%-20 \%$. The right plot presents the same for the homogenization and inverse problem approaches, along with the values computed using the Maxwell-Garnett mixing formula and a weighted average.

The Cole-Cole diagrams corresponding to the complex permittivities shown in Figures 10 and 11 are shown in the left and right plots of Figure 12.

The Cole-Cole diagram for a Debye model is a semi-circle and the peaks correspond to the relaxation frequency. As both the weighted average values and the inverse problem approach use the Debye model, their diagrams are semi-circles. Both the Maxwell-Garnett rule and the homogenization method have more complex Cole-Cole diagrams as evidenced by the bend in the 
curves. This discrepancy from the Debye model is more pronounced at the lower frequencies for this volume fraction.

\section{2 $50 \%-50 \%$ Volume Fraction}

The left plot of Figure 13 depicts the DRF's for the inverse problem result, the solution from the homogenization approach, as well as the curves for a Debye model of pure water and for pure ethanol. In the right plot of Figure 13 we compare the DRF from the inverse problem and homogenization approaches to the MG mixing rule result and the DRF obtained by using the weighted average of values in the Debye model. The inverse problem DRF is very similar to that of the weighted averages; however, the difference between the corresponding outputs from the interrogation simulations was clearly seen in Figure 7.
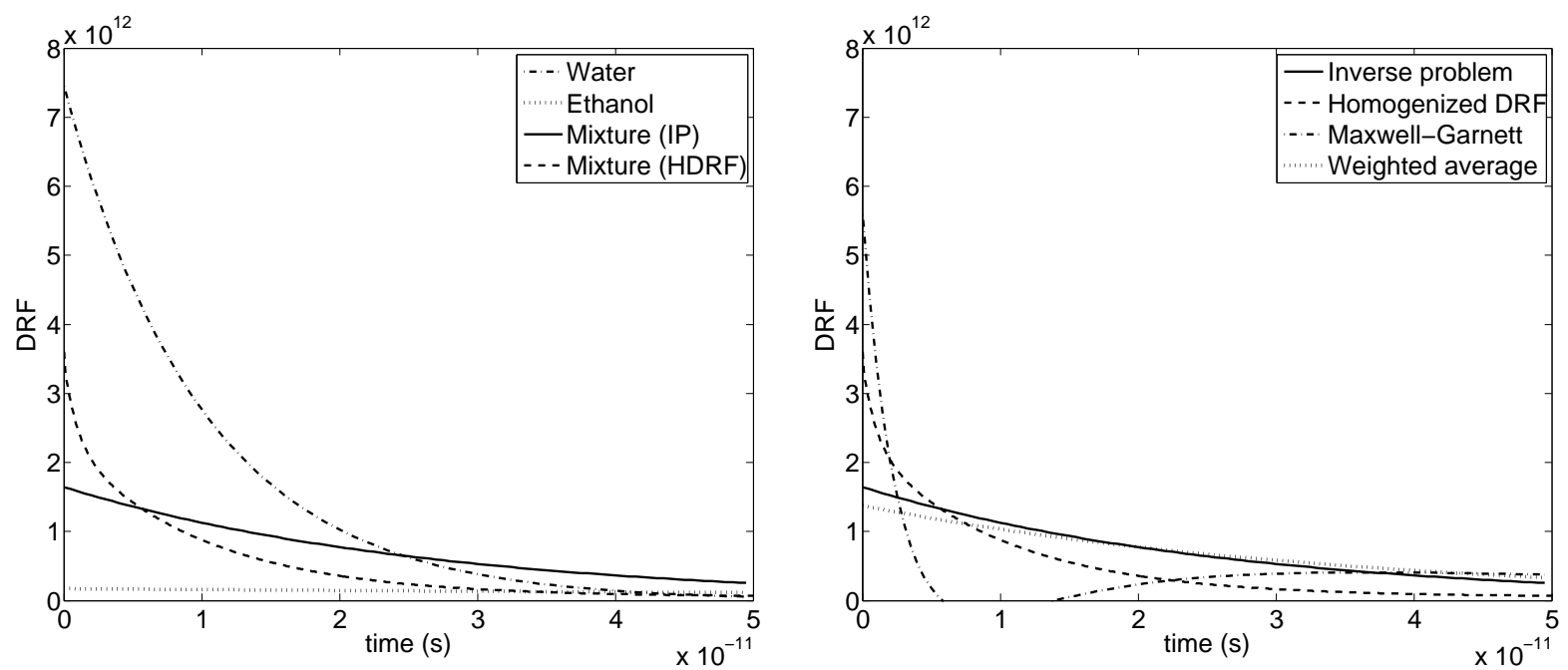

Figure 13: The left side presents a plot of the DRF resulting from the homogenization approach and the inverse problem approach, along with the plots for the Debye model of water and ethanol, using a volume fraction of $50 \%-50 \%$. The right side depicts the same for the homogenization and inverse problem approaches, along with the DRF's computed using the Maxwell-Garnett mixing formula and a weighted average. The DRF of the weighted average approach and the inverse problem method are nearly indistinguishable.

The real and imaginary parts of the permittivity resulting from the homogenization approach and the inverse problem approach, are plotted in Figure 14, along with the values for pure water and pure ethanol. In Figure 15 the real and imaginary parts of the permittivity from the homogenization approach and the inverse problem approach are compared to the values resulting from taking the weighted average of parameters and the Maxwell-Garnett mixing method.

The peaks in the plots of the imaginary parts denote the frequency at which the maximum attenuation of the field in the material occurs, (in the Debye case it is known as the relaxation frequency). Note that both the Maxwell-Garnett and the homogenization methods still have peaks relatively close to that of water, as compared to ethanol, considering that it is a $50 \%-50 \%$ mixture. Both the parameter estimation method and the approach using weighted average of parameters 

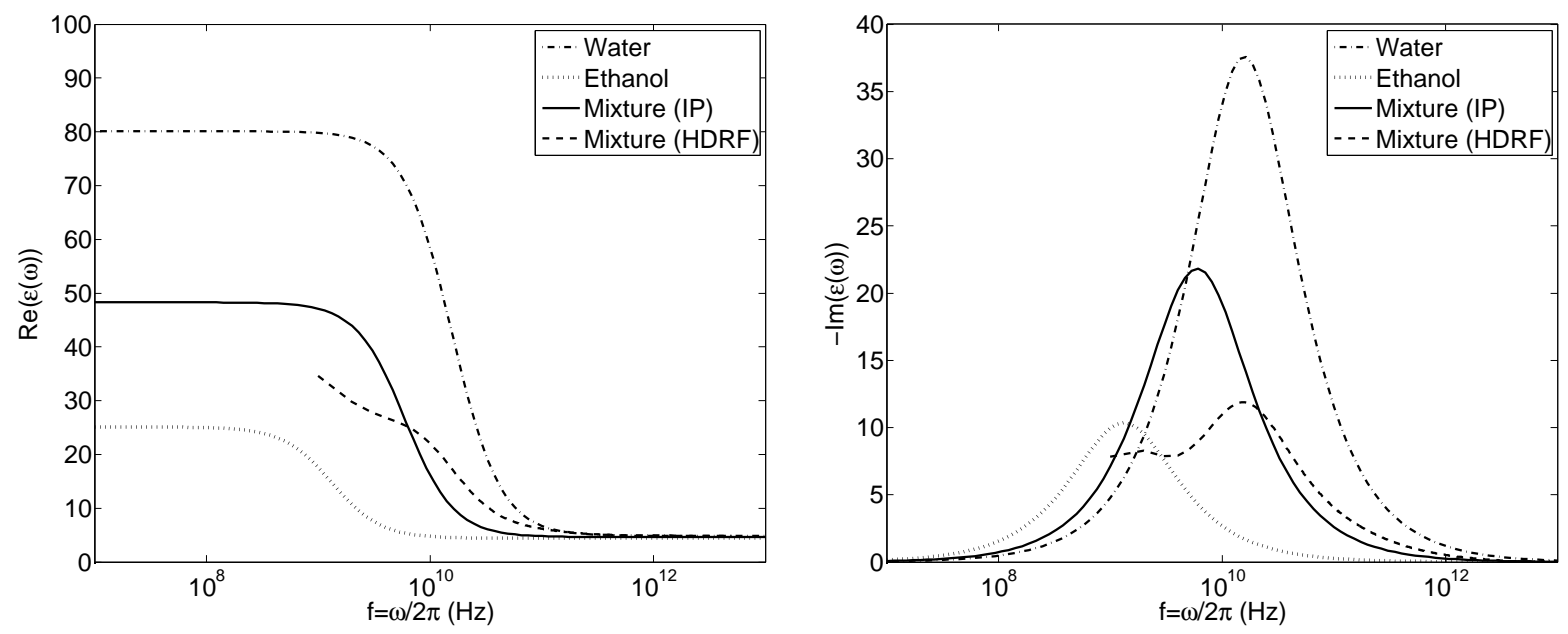

Figure 14: The left (right) graph depicts the real (negative imaginary) part of the permittivity resulting from the homogenized DRF and the inverse problem approach, using a volume fraction of $50 \%-50 \%$, along with the values for pure water and pure ethanol.
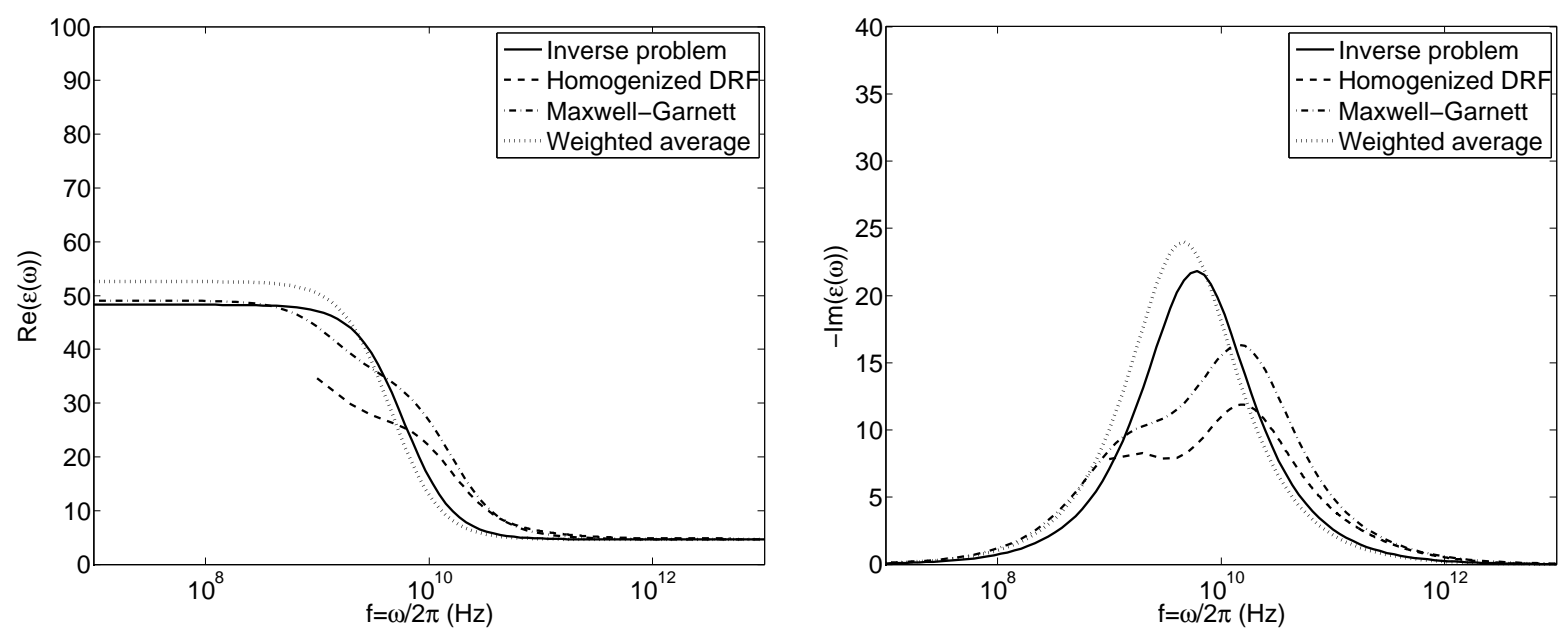

Figure 15: The left (right) graph presents the real (negative imaginary) part of the permittivity resulting from the homogenized DRF and the inverse problem approach, along with the values computed using a weighted average and the Maxwell-Garnett mixing formula, using a volume fraction of $50 \%-50 \%$.

result in peaks very close to the average of the relaxation frequencies of water and ethanol.

The Cole-Cole diagrams corresponding to the complex permittivities shown in Figures 14 and 15 are shown in the left and right plots of Figure 16. As both the weighted average values and the inverse problem approach use the Debye model, their diagrams are again semi-circles. The Maxwell-Garnett rule and the homogenization method are again more complicated, and have an even more pronounced bend in the curve than the $80 \%-20 \%$ case. The frequency at which is occurs is also closer to the peak frequency than the previous case. 

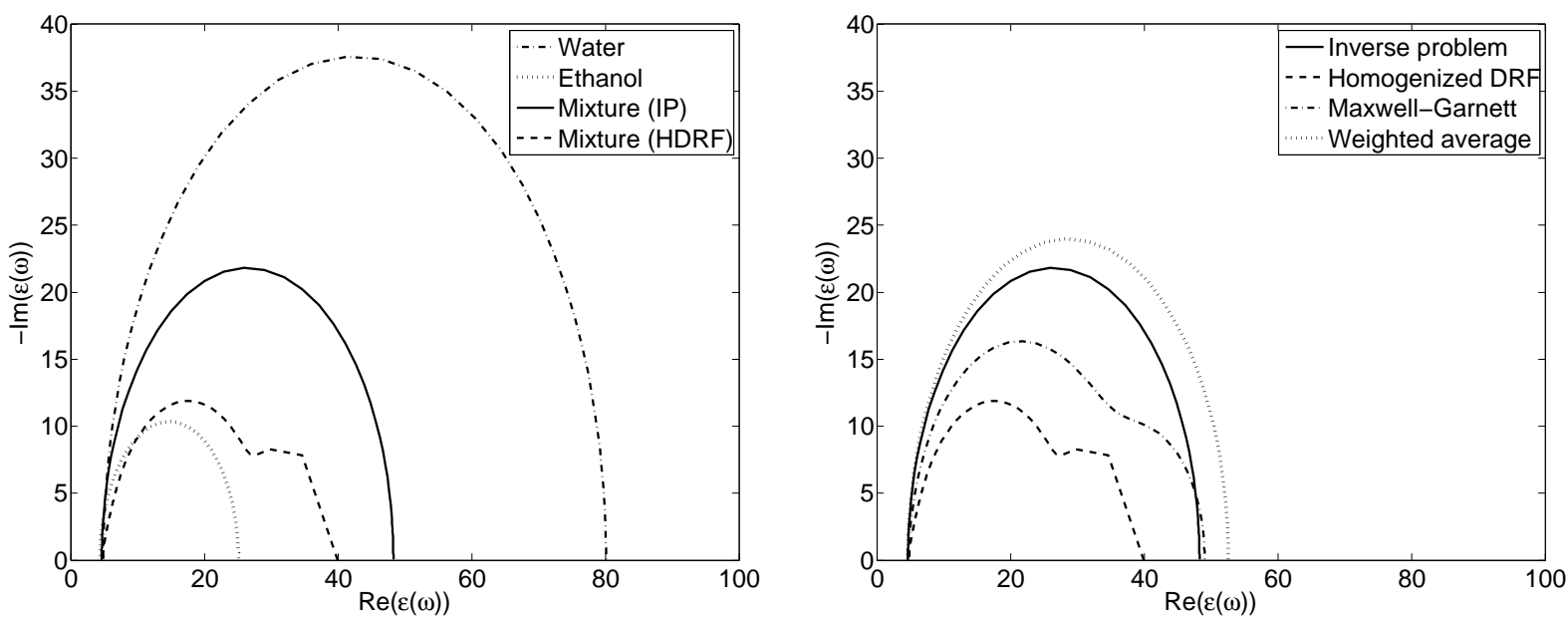

Figure 16: The left plot depicts the Cole-Cole diagram of the complex permittivity resulting from the homogenization approach and the inverse problem approach, along with the values from the Debye model for water and ethanol, using a volume fraction of $50 \%-50 \%$. The right plot presents the same for the homogenization and inverse problem approaches, along with the values computed using the Maxwell-Garnett mixing formula and a weighted average.

\section{$6.320 \%-80 \%$ Volume Fraction}

The left plot of Figure 17 depicts the DRF's for the inverse problem result, the solution from the homogenization approach, as well as the curves for a Debye model of pure water and for pure ethanol. In the right plot of Figure 17 we compare the DRF from the inverse problem and homogenization approaches to the MG mixing rule result and the DRF obtained by using the weighted average of values in the Debye model. The inverse problem DRF is very similar to that of the weighted averages; however, the difference between the corresponding outputs from the interrogation simulations was clearly seen in Figure 8.

The real and imaginary parts of the permittivity resulting from the homogenization approach and the inverse problem approach, are plotted in Figure 18, along with the values for pure water and pure ethanol. In Figure 19 the real and imaginary parts of the permittivity from the homogenization approach and the inverse problem approach are compared to the values resulting from taking the weighted average of parameters and the Maxwell-Garnett mixing method.

The peaks in the plots of the imaginary parts denote the frequency at which the maximum attenuation of the field in the material occurs, (in the Debye case it is known as the relaxation frequency). Note that both the Maxwell-Garnett and the homogenization methods still have peaks relatively close to that of water, as compared to ethanol, considering that it is a $20 \%-80 \%$ mixture. Both the parameter estimation method and the approach using weighted average of parameters result in peaks very close to the average of the relaxation frequencies of water and ethanol.

The Cole-Cole diagrams corresponding to the complex permittivities shown in Figures 18 and 19 are shown in the left and right plots of Figure 20. As both the weighted average values and the inverse problem approach use the Debye model, their diagrams are again semi-circles. The Maxwell-Garnett rule and the homogenization method are again more complicated, and have an 

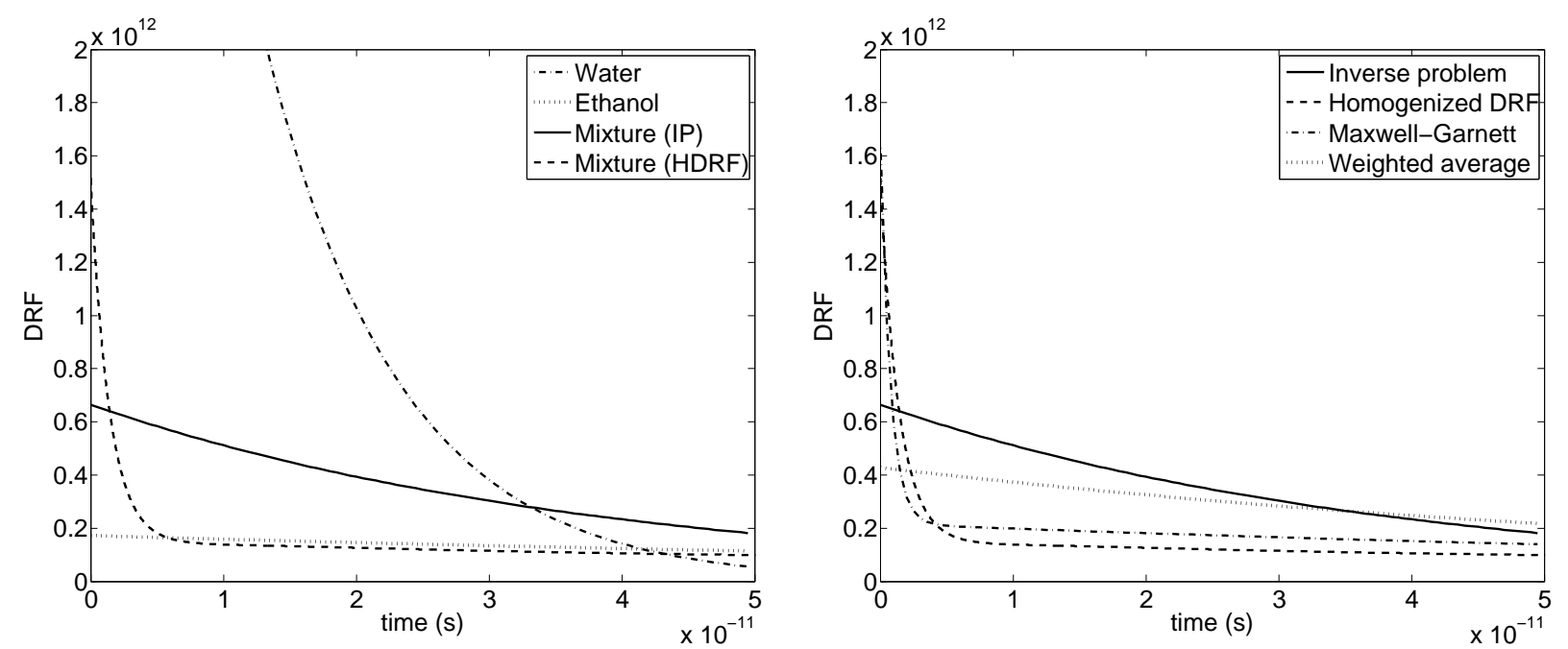

Figure 17: The left side presents a plot of the DRF resulting from the homogenization approach and the inverse problem approach, along with the plots for the Debye model of water and ethanol, using a volume fraction of $20 \%-80 \%$. The right side depicts the same for the homogenization and inverse problem approaches, along with the DRF's computed using the Maxwell-Garnett mixing formula and a weighted average. The DRF of the weighted average approach and the inverse problem method are nearly indistinguishable.
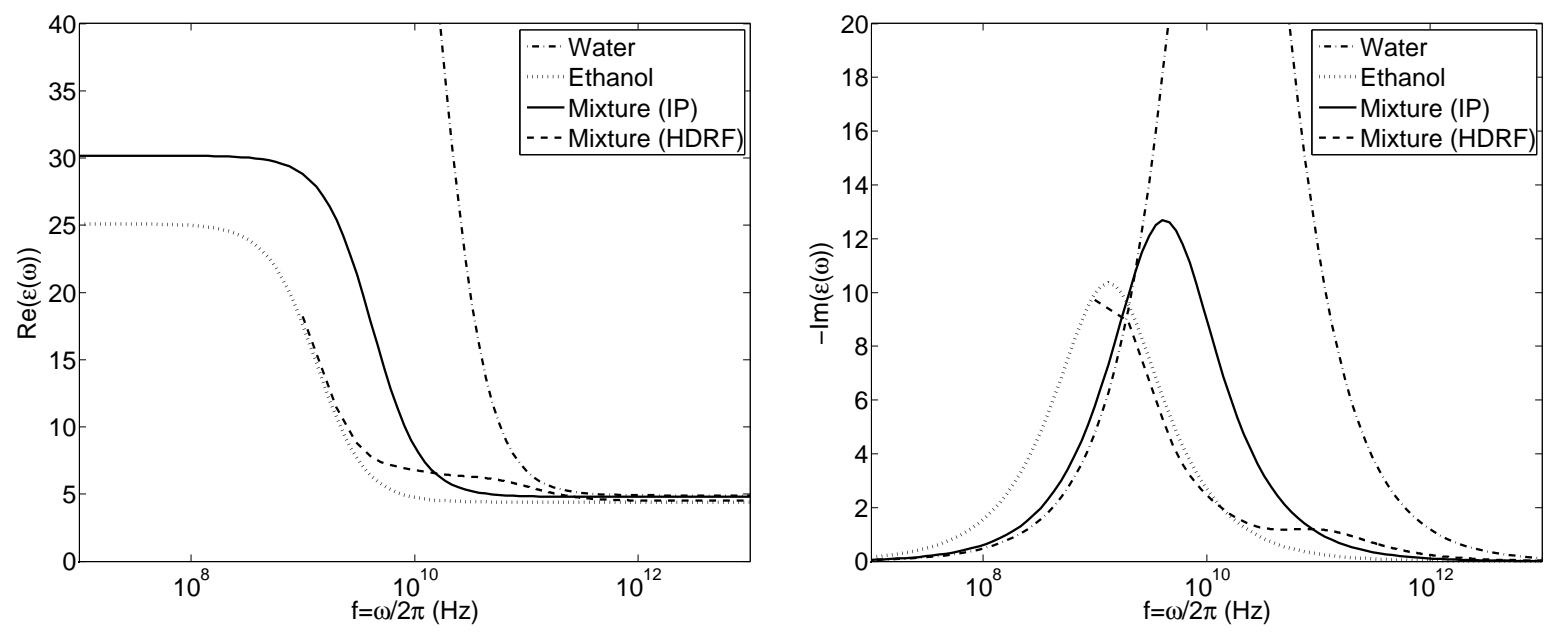

Figure 18: The left (right) graph depicts the real (negative imaginary) part of the permittivity resulting from the homogenized DRF and the inverse problem approach, using a volume fraction of $20 \%-80 \%$, along with the values for pure water and pure ethanol.

even more pronounced bend in the curve than the $80 \%-20 \%$ case. The frequency at which is occurs is also closer to the peak frequency than the previous case. 

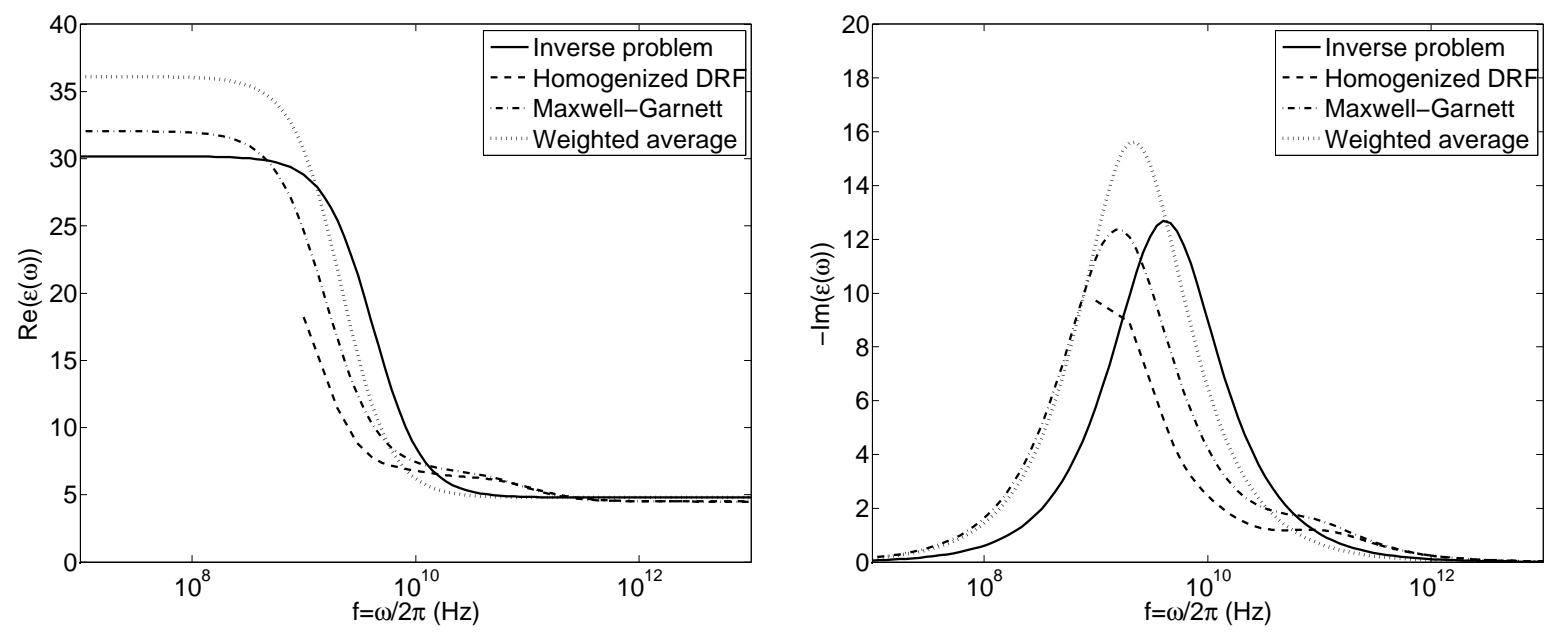

Figure 19: The left (right) graph presents the real (negative imaginary) part of the permittivity resulting from the homogenized DRF and the inverse problem approach, along with the values computed using a weighted average and the Maxwell-Garnett mixing formula, using a volume fraction of $20 \%-80 \%$.
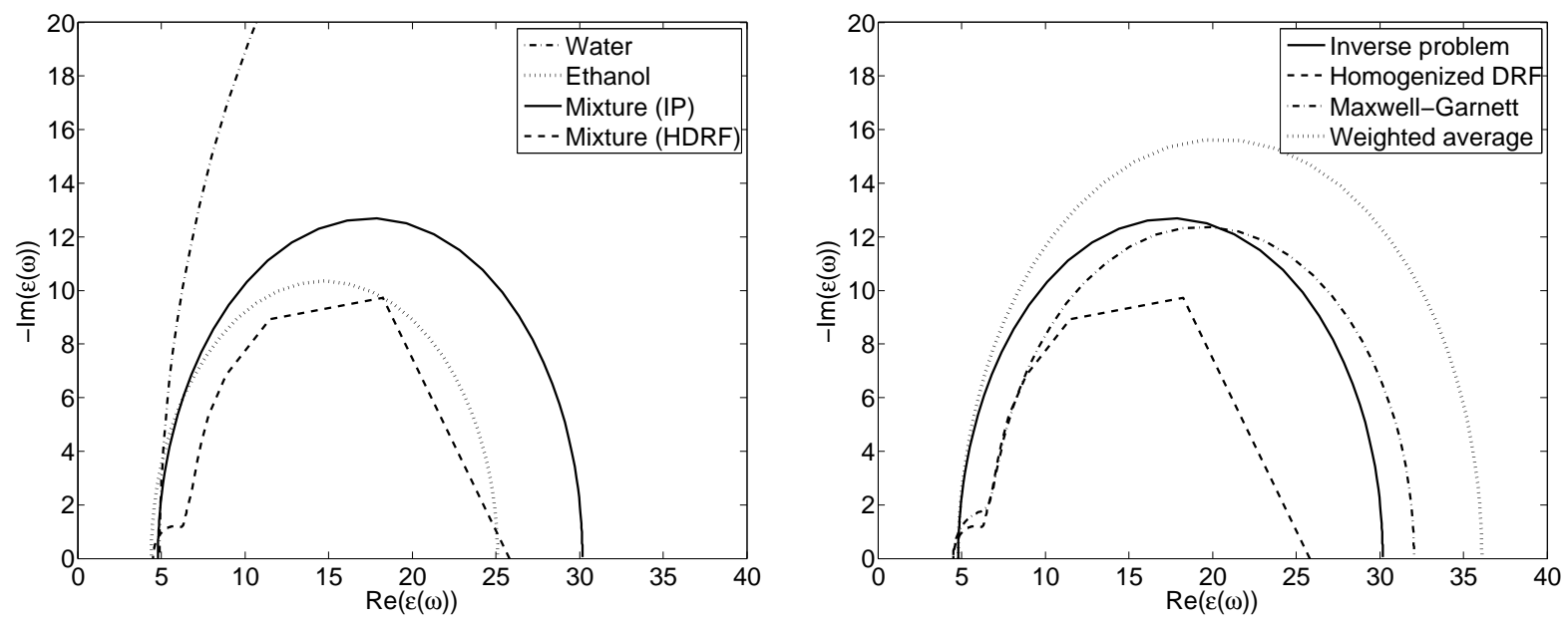

Figure 20: The left plot depicts the Cole-Cole diagram of the complex permittivity resulting from the homogenization approach and the inverse problem approach, along with the values from the Debye model for water and ethanol, using a volume fraction of $20 \%-80 \%$. The right plot presents the same for the homogenization and inverse problem approaches, along with the values computed using the Maxwell-Garnett mixing formula and a weighted average.

\section{Convergence of DFT for Homogenization DRF}

In order to display the complex permittivity resulting from the DRF computed with the homogenization approach, one must take the DFT of the DRF at many time points. Further, in order for the maximum frequency component to be large enough to display useful information, the time step must be very small. For example, in order to compute the complex permittivity up to $f=5 \times 10^{12}$ 
$\mathrm{Hz}$ (where the real part of the permittivity converges to $\epsilon_{\infty}$ ), the time step for calculating the DRF in the homogenization method must be $10^{-13}$ seconds. Further, for sufficient resolution down to $f=10^{8} \mathrm{~Hz}$ (where the real part of the permittivity converges to $\epsilon_{s}$ ), the number of time steps must be greater than 20000. In our numerical experiments, each time step takes on the order of .1 seconds, as a linear system for the finite element method must be solved. The following subsections show computations of the DFT for various DRF's computed with successively smaller time steps.

\section{$7.1 \quad 80 \%-20 \%$ Volume Fraction}

We compute the homogenized DRF for the volume fraction $80 \%-20 \%$ water-ethanol using time steps $d t=\left\{10^{-14}, 10^{-13}, 10^{-12}\right\}$. The resulting curves are shown in the left plot of Figure 21 . The convergence in the time domain is quickly apparent, however, many more steps would be required for full convergence of the frequency domain results, as seen in the right plot which contains the Cole-Cole diagram for each time step. The real and imaginary parts of the permittivity resulting
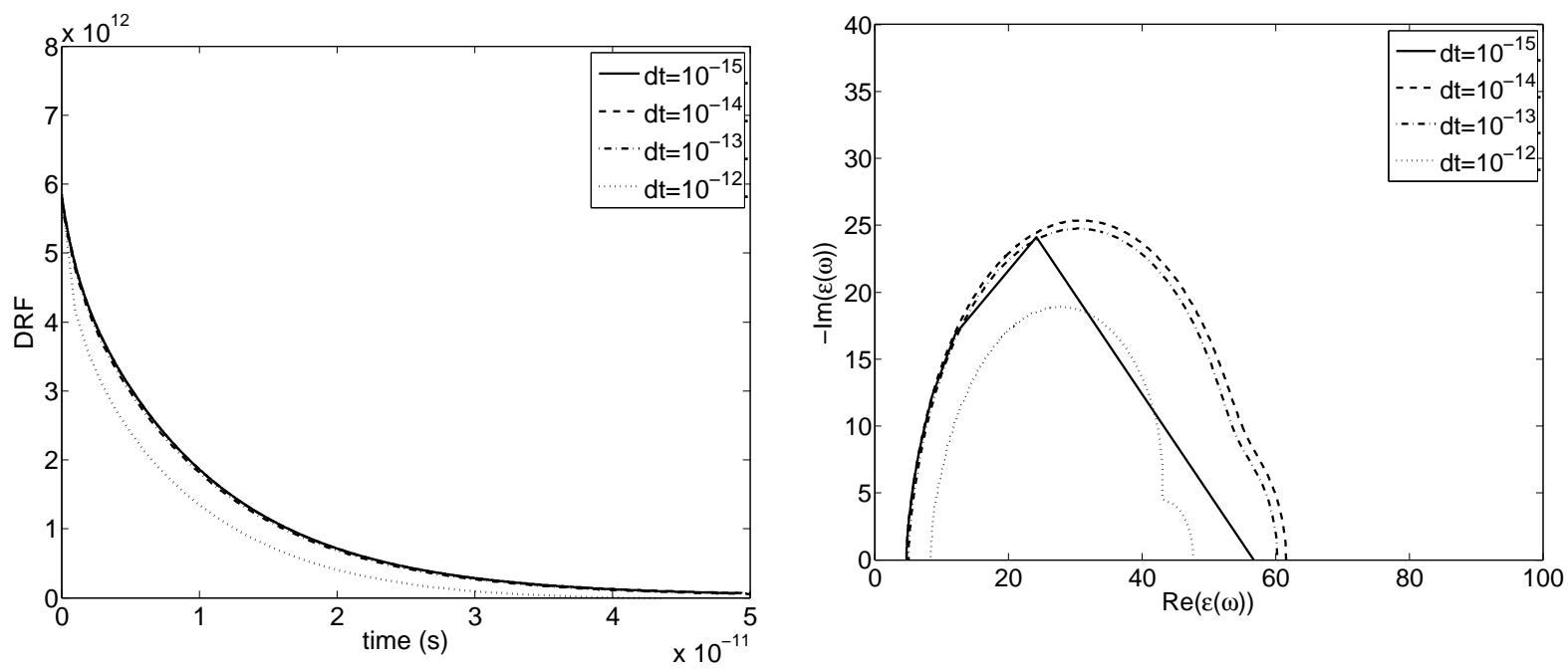

Figure 21: The left side presents a plot of the DRF resulting from the homogenization approach using time steps $d t=\left\{10^{-14}, 10^{-13}, 10^{-12}\right\}$. for the case of an $80 \%-20 \%$ volume fraction of water and ethanol. The right plot depicts the Cole-Cole diagram of the complex permittivity resulting from the homogenization approach for each time step. The incompleteness is due to lack of sufficient time steps. $10^{5}$ steps are shown.

from the homogenization approach using time steps $d t=\left\{10^{-14}, 10^{-13}, 10^{-12}\right\}$ are plotted in Figure 22. The high frequency part of the curves are complete and appear to be converging to a limiting effective complex permittivity.

\section{$7.2 \quad 20 \%-80 \%$ Volume Fraction}

For the case of a volume fraction of $20 \%-80 \%$ water-ethanol we use the time steps $d t=\left\{10^{-16}\right.$, $\left.10^{-14}, 10^{-13}\right\}$. The resulting curves are shown in the left plot of Figure 23. Although fewer time steps were computed in the $d t=10^{-16}$ case, clear agreement in the time domain is reached with 

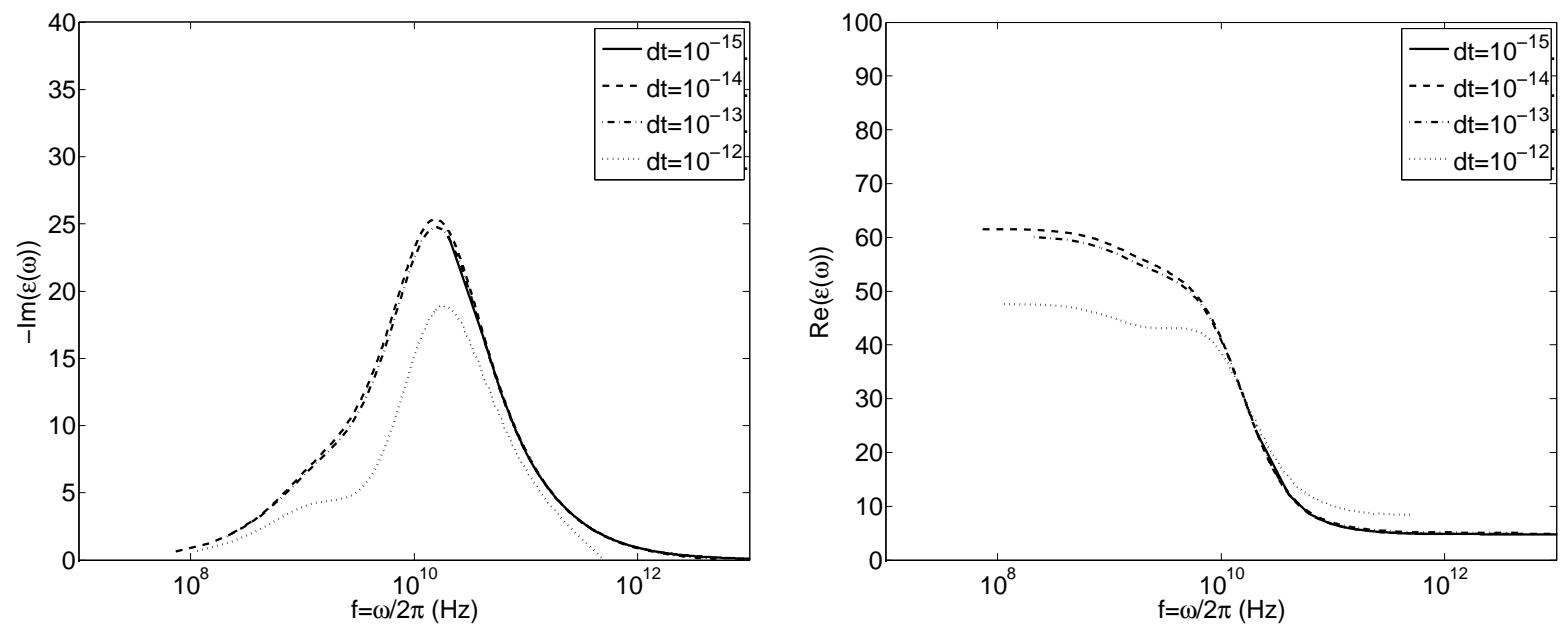

Figure 22: The left (right) graph depicts the real (negative imaginary) part of the permittivity resulting from the DFT of the homogenized DRF, using a volume fraction of $80 \%-20 \%$, computed with time steps $d t=\left\{10^{-14}, 10^{-13}, 10^{-12}\right\}$.

only $d t=10^{-14}$. The right plot of Figure 23 depicts the Cole-Cole diagram for each time step. All three resolutions agree at the higher frequencies (the left side of the plot), which is where the effect of the composite material is most obvious. The overlapping circles is characteristic of mixtures of Debye substances, and the diagrams here agree nicely with the classical Maxwell-Garnett mixing rule shown in Figure 20. The real and imaginary parts of the permittivity resulting from the
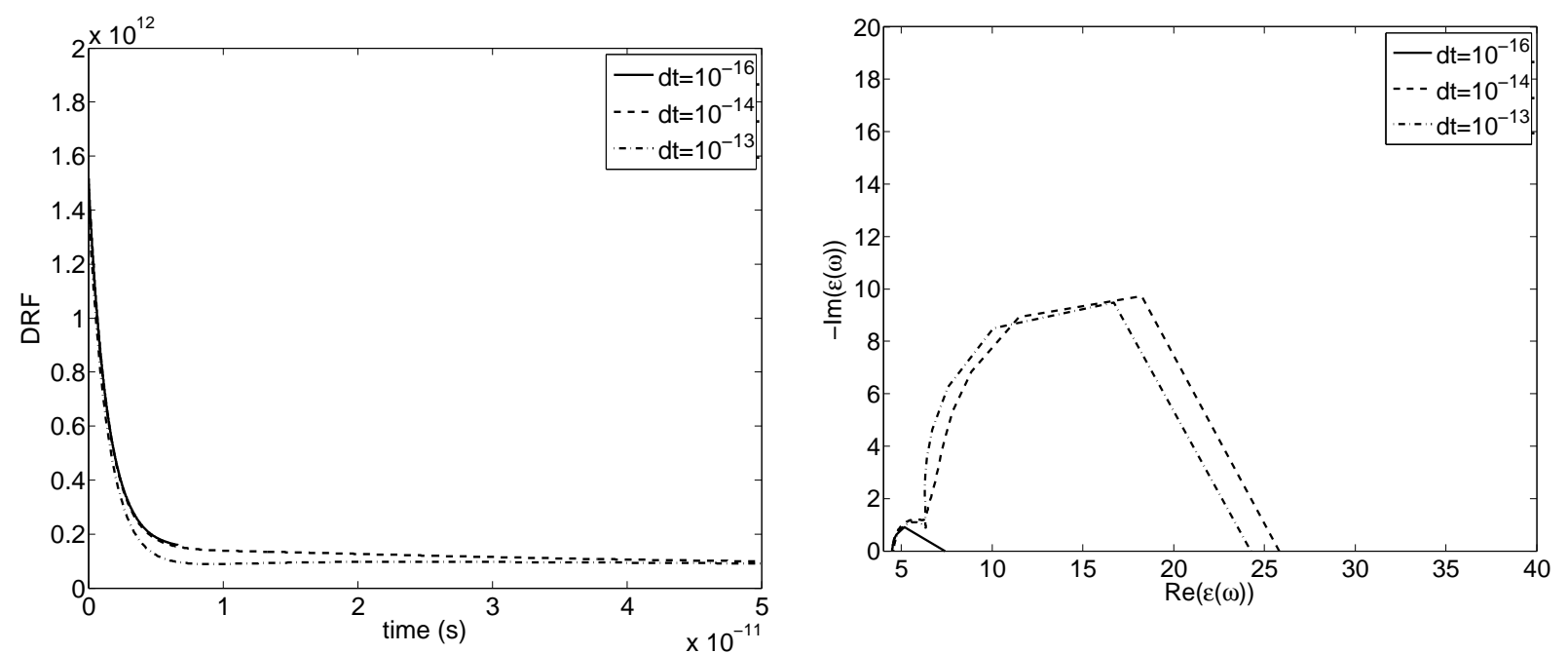

Figure 23: The left side presents a plot of the DRF resulting from the homogenization approach using time steps $d t=\left\{10^{-16}, 10^{-14}, 10^{-13}\right\}$. for the case of an $20 \%-80 \%$ volume fraction of water and ethanol. The right plot depicts the Cole-Cole diagram of the complex permittivity resulting from the homogenization approach for each time step. The inaccuracies due to the incompleteness of the time domain DRF are clearly apparent.

homogenization approach using time steps $d t=\left\{10^{-16}, 10^{-14}, 10^{-13}\right\}$, are plotted in Figure 24 . 
The high frequency part of the curves appear to coincide better than for the $80 \%-20 \%$ case, however the lower frequencies are not sufficiently resolved to be able to estimate the effective static permittivity $\epsilon_{s}$.
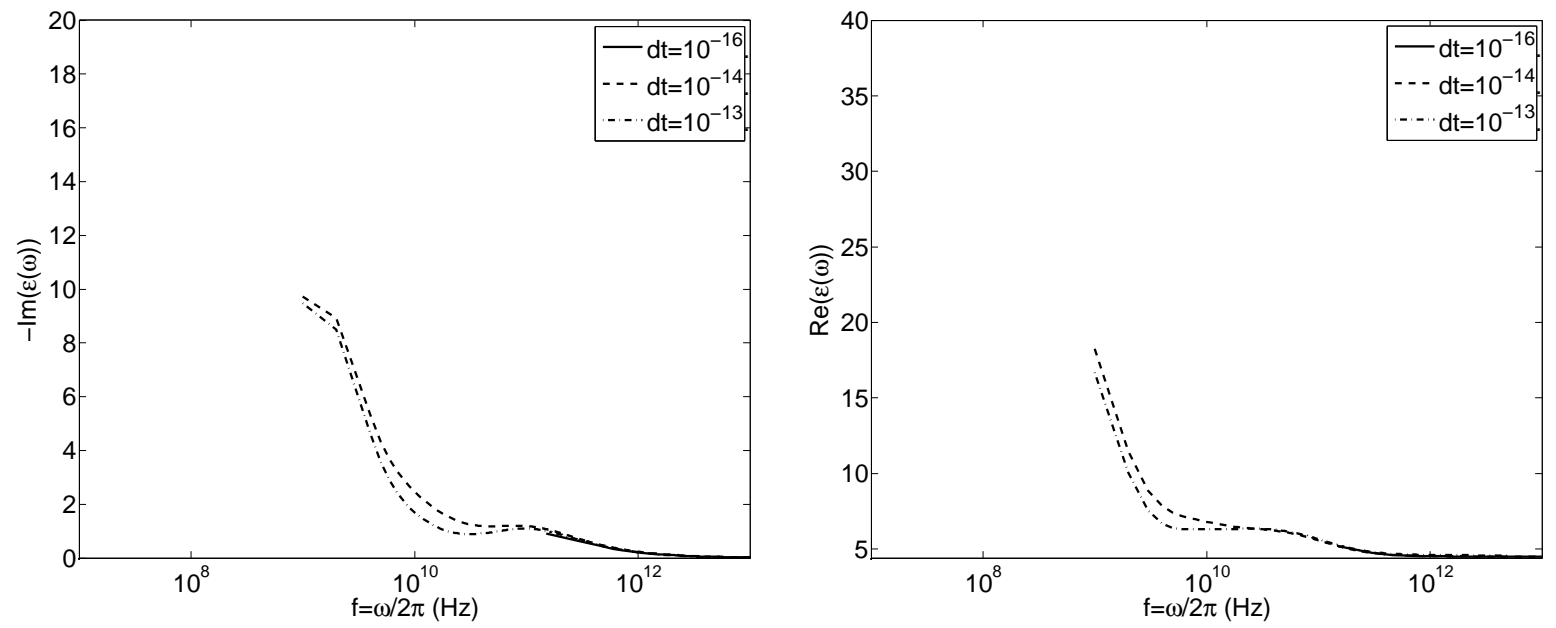

Figure 24: The left (right) graph depicts the real (negative imaginary) part of the permittivity resulting from the DFT of the homogenized DRF, using a volume fraction of $20 \%-80 \%$, computed with time steps $d t=\left\{10^{-16}, 10^{-14}, 10^{-13}\right\}$.

\section{Conclusions}

The Maxwell-Garnett mixing rule is only valid for spherical or circular inclusions (ellipsoidal inclusions can also be considered). The periodic unfolding homogenization method allows for any two or three dimensional geometry. It is important to note, however, that the results of all homogenization techniques (including $\mathrm{MG}$ ) are valid when the size of the inclusion are small in comparison with the wave length of the sources and fields. For higher frequencies scattering effects are no longer negligible. A second advantage of the homogenization method presented here is the flexibility it affords in assumptions about material polarization laws. In this paper we have computed the DRF for a Debye-Debye mixture using a recursive convolution method. The recursive convolution approach can be extended to the cases of mixtures involving Lorentz or higher order dispersive media in which the DRF is exponential in nature.

A mixture of materials, which may each be described by the same simple model, in general will not be accurately described by that same model. For example, a Debye-Debye mixture has an effective complex permittivity which is not of Debye form, nor even of Lorentz form. The homogenization methods described here attempt to capture this complexity. However, for certain applications it is not necessary to have a high level of accuracy for those frequencies which do not appear in the problem, especially if the added complexity necessary for increased accuracy is costprohibitive. For example, in a simulation with an interrogating signal of narrow frequency band, the complex permittivity need only be accurate in those frequencies. Using an inverse problem formulation designed around the specific fields of interest can give a cost-effective method for modeling complex materials with relatively simple formulas. 
As mentioned earlier, for both the homogenization method based on periodic unfolding as well as the Maxwell-Garnett model, knowledge of the volume proportions of the components of the mixture is needed in order to calculate effective parameters of this composite dielectric. However, the parameter estimation method is capable of using experimental data in the inverse problem, in which case there would be no assumptions needed on the volume fraction or dielectric parameters except perhaps in formulating a sufficiently accurate initial value for faster convergence of the optimization routine. In generating synthetic data however, one must of course have the volume proportions, as well as dielectric parameters for each material, including possibly distributions of parameters. While there is a large initial investment in computational time to perform the many forward solves needed to determine the optimal parameter set, having effective parameters for a simplified model which closely matches the data of interest may lead to significant savings in later simulations such as in geometric inverse problems involving crack or flaw detection. One of the biggest advantages to using the inverse problem parameter estimation approach is that it may be tailored to specific applications with narrow frequency bands such as those that often occur in the fields of non-destructive evaluation (NDE) or remote sensing.

\section{Acknowledgments}

This research was supported in part by the U. S. Air Force Office of Scientific Research under grant AFOSR FA9550-04-1-0220 and in part by the National Institute of Aerospace (NIA) and NASA under grant NIA/NCSU-03-01-2536-NC.

\section{References}

[1] H. T. Banks, V. A. Bokil, D. Cioranescu, N. L. Gibson, G. Griso, and B. Miara, Homogenization of periodically varying coefficients in electromagnetic materials, J. Sci. Comput., available online: DOI10.1007/s10915-006-9091-y; to appear in print.

[2] H. T. Banks, V. A. Bokil, and N. L. Gibson, Analysis of stability and dispersion in a finite element method for Debye and Lorentz dispersive media, Center for Research in Scientific Computation Tech Rept CRSC-TR06-21, August, 2006.

[3] H. T. Banks, M. W. Buksas, and T. Lin, Electromagnetic Material Interrogation Using Conductive Interfaces and Acoustic Wavefronts, Frontiers in Applied Mathematics, v. FR21, SIAM, Philadelphia, PA, 2000.

[4] H. T. Banks and N. L. Gibson, Electromagnetic inverse problems involving distributions of dielectric mechanisms and parameters, Center for Research in Scientific Computation Tech Rept CRSC-TR05-29, August, 2005; Quarterly of Applied Mathematics, to appear.

[5] H. T. Banks, N. L. Gibson, and W. P. Winfree, Gap detection with electromagnetic Terahertz signals, Nonlinear Analysis: Real World Applications, 6 (2005), 381-416.

[6] A. Bossavit, G. Griso, and B. Miara, Modeling of periodic electromagnetic structures bianisotropic materials with memory effects, J. Math. Pures Appl., 84 (2005), 819-850. 
[7] C. J. F. Böttcher and P. Bordewijk, Theory of Electric Polarization,Vol. II, Elsevier, New York, 1978.

[8] D. Cioranescu, A. Damlamian, and G. Griso, Periodic unfolding and homogenization, C. $R$. Acad. Sci. Paris, Ser. I, 335 (2002), 99-104.

[9] K.S. Cole and R.H. Cole, Dispersion and absorption in dielectrics, J. Chemical Phys. 9 (1941), $341-351$.

[10] P. Debye, Polar Molecules, Chemical Catalog Co., New York, 1929.

[11] K.R. Foster and H.P. Schwan, Dielectric properties of tissues and biological materials: A critical review, Critical Rev. in Biomed. Engr. 17 (1989), 25-104.

[12] D. F. Kelley and R. J. Luebbers, Piecewise linear recursive convolution for dispersive media using FDTD, IEEE Trans. Antennas Propagat., 44 (1996), 792-797.

[13] R. J. Luebbers, F.P. Hunsberger, K. S. Kunz, R. B. Standler, and M. Schneider, A frequencydependent finite difference time-domain formulation for dispersive materials, IEEE Trans. Elect. Compat., 32 (1990), 222-227.

[14] G. Kristensson, S. Rikte and A. Sihvola, Mixing formulas in the time domain, J. Opt. Soc. Am. A, 15 (1998), 1411-1422.

[15] J.C.M. Garnett, Colours in metal glasses and in metal films, Philos. Trans. R. Soc. London Ser. A, 203 (1904), 385-420. 